\title{
Molecular Tracking of the Leishmania Parasite
}

\author{
Srija Moulik, Shilpa Sengupta and Mitali Chatterjee* \\ Department of Pharmacology, Institute of Postgraduate Medical Education and Research, Kolkata, India
}

With the Visceral Leishmaniasis/Kala-azar Elimination Program in South Asia in its consolidation phase, the focus is mainly on case detection, vector control, and identifying potential sources of infection. Accordingly, emphasis is presently on curbing transmission, which is potentially achievable by identification and elimination of potential reservoirs. The strongest contenders for being the disease reservoir are cases of Post Kala-azar Dermal Leishmaniasis (PKDL) which occurs in a minor proportion of individuals apparently cured of Visceral Leishmaniasis (VL). The demonstration of parasites in tissue aspirates despite being a risky and invasive process is the gold standard for diagnosis of $\mathrm{VL}$, but is now being replaced by serological tests e.g., rK39 strip test and direct

OPEN ACCESS

Edited by:

Om Prakash Singh,

Banaras Hindu University, India

Reviewed by:

Manju Jain,

Central University of Punjab, India Isabel Mauricio,

New University of Lisbon, Portugal

*Correspondence:

Mitali Chatterjee ilatimc@gmail.com

Specialty section:

This article was submitted to

Parasite and Host,

a section of the journal

Frontiers in Cellular and

Infection Microbiology

Received: 30 October 2020 Accepted: 06 January 2021

Published: 22 February 2021

Citation:

Moulik S, Sengupta S and Chatterjee M (2021) Molecular Tracking of the Leishmania Parasite. Front. Cell. Infect. Microbiol. 11:623437.

doi: 10.3389/fcimb.2021.623437 agglutination test. However, these antibody based tests are limited in their ability to diagnose relapses, detect cases of PKDL, and monitor effectiveness of treatment. Accordingly, detection of antigen or nucleic acids by polymerase chain reaction has been successfully applied for monitoring of parasite kinetics. This review article provides updated information on recent developments regarding the available antibody or antigen/ nucleic acid based biomarkers for longitudinal monitoring of patients with VL or PKDL and emphasizes the need for availability of studies pertaining to quantification of treatment response or relapse.

Keywords: anti-leishmanial antibodies, biomarkers, HIV-VL, kinetoplast DNA kDNA, molecular diagnosis, Post Kala-azar Dermal Leishmaniasis PKDL, Visceral Leishmaniasis VL

\section{INTRODUCTION}

Worldwide, an estimated 50,000 to 90,000 new cases of kala-azar or Visceral Leishmaniasis (VL), caused by the parasite Leishmania donovani occur annually, with the contribution of Bangladesh, India, and Nepal being around 67\% (https://www.who.int/news-room/fact-sheets/detail/ Leishmaniasis last accessed on 4th December, 2020). In a joint VL elimination initiative launched by the Governments of India, Bangladesh, and Nepal in 2005, the target for elimination by 2020 was aimed at reduction of the annual incidence of VL to below 1/10,000 persons at an upazilla level in Bangladesh, sub-districts [(block public health centre (PHC)] level in India and district level in Nepal (Sundar et al., 2018). This elimination was considered feasible owing to the defined and limited geographical spread of VL, absence of an animal reservoir, a single vector Phlebotomus argentipes, availability of an effective diagnostic test, use of an oral drug miltefosine which was later replaced by a 
single dose of liposomal amphotericin B (AmBisome), along with a strong political commitment (Hirve et al., 2017; Alves et al., 2018; Gedda et al., 2020).

The Kala Azar Elimination Programme (KAEP) consists of four consecutive phases and began with a "preparatory phase" which involved development/review of national policy, strategic and advocacy plans, operational plans to implement the national plan for elimination, development, and adoption of technical guidelines (Sundar et al., 2018). This was followed by a multiprolonged "attack phase" that included integrated vector management with indoor residual spraying for 5 years in affected areas along with active surveillance, early diagnosis and complete treatment. Till date, the number of VL cases in India, Nepal, and Bangladesh have declined steadily from over 77,000 reported cases in 1992 to fewer than 7,000 cases in 2016 (Rijal et al., 2019) and further reduced to 3,128 in 2019 (World Health Organization, 2020).

The KAEP has since moved into the consolidation phase, where ongoing active surveillance is aimed at detecting and treating potential disease reservoirs, namely asymptomatic cases of VL and patients with Post-kala-azar dermal Leishmaniasis (PKDL), the latter being a dermal aftermath in individuals apparently cured of VL. This consolidation phase will end when three years of active surveillance demonstrates no increase in the incidence rate at district/subdistrict/upazila levels in the endemic countries. Finally, a maintenance phase will be undertaken to ensure the case incidence is sustained at less than 1 per 10,000 population (World Health Organization, 2020).

As PKDL cases harbor parasites in dermal lesions that are easily accessible to the sandfly, it makes them key players in the transmission cycle, and bears the burden of being a major factor potentially capable of jeopardising the success of the KAEP (Duthie et al., 2019). However, tracking patients with PKDL is a formidable challenge owing to its low morbidity and practically no mortality. This accounts for their poor health seeking behavior, and ultimately translates into PKDL being a potent, mobile reservoir (Zijlstra et al., 2017; Burza et al., 2018). Furthermore, identifying biomarkers in PKDL is hampered by the lack of adequate studies along with a considerable heterogeneity, reiterating the need for well-designed trials to assess diagnostic accuracy (Adams et al., 2013). In addition, as the pathophysiology of PKDL is different from VL or other cutaneous leishmaniases and even differs based on geographical locations, one cannot extrapolate from existing biomarkers of Leishmania infection (Kip et al., 2015; Zijlstra et al., 2017).

With the availability of effective diagnostic tools and treatments for VL and PKDL cases as also integrated vector management, countries have progressed towards the elimination goal. A lingering concern is that this lowering of case numbers can lead to a decreasing awareness within the communities and health care providers, and result in cases of VL and PKDL being ignored or missed, resulting in resurgence. It is important that such a scenario be averted, emphasizing the need for objective quantification of the infection burden. The availability of molecular based tools could facilitate development of rapid and high throughput approaches to detect parasites. Accordingly, this review focuses on the recent developments regarding antibody or antigen/nucleic acid based biomarkers with potential for longitudinal monitoring of patients with VL or PKDL, and highlights the limited availability of studies pertaining to quantification of treatment response and/or relapse.

\section{Ethics}

Written informed consent was obtained from the individual(s) and/or minor(s)' legal guardian/next of kin for the publication of any potentially identifiable images or data included in this article.

\section{Strategy for Literature Search}

The potential biomarkers for VL and PKDL were identified via the PubMed database pertaining to publications after 2000 and restricted to the English language, using the following key words: " $(((V$ isceral Leishmaniasis-[title]) or Kala -azar[title]) or PKDL [title]) and $((((()(($ biomarker $)$ or biomarkers) or marker $)$ or diagnosis) or markers) or level) or levels) or concentration) or activity) or profile) or Leishmania antigen/antibody based test)". The date last searched was $30^{\text {th }}$ September, 2020 and publications that did not focus on the identification or evaluation of biomarkers as a diagnostic approach were excluded (Figure 1). Additionally, secondary literature was included based on references included in the identified primary literature.

\section{Evaluation Criteria}

The translational potential of serological and nucleic acid biomarkers was based on five criteria: (i) Assay sensitivity, the marker's quantification in patients at disease presentation (ii) specificity, in relation to co-endemic infectious diseases, such as malaria, tuberculosis, enteric fever and HIV for VL, and in case of PKDL, diseases like leprosy, psoriasis and vitiligo, (iii) advantages, (iv) limitations/disadvantages, and (v) ability to monitor responses to anti-leishmanial therapy

\section{RESULTS AND DISCUSSION}

\section{Literature Search}

The primary literature search identified 264 studies for which the titles were screened and assessed for relevance based on key words “(((Leishmaniasis-[title]) or Kala -azar[title]) or PKDL [title] $)$ and $(((((()($ biomarker $)$ or biomarkers $)$ or marker $)$ or diagnosis) or markers) or level) or levels) or concentration) or activity) or profile) or PCR) or qPCR)", Figure 1. Abstracts ( $=$ 210) were considered as non-relevant (Figure 1) and thereafter, full texts of the remaining studies were assessed for their relevance. This translated into 54 publications being included in this review. Additionally, 35 more studies were identified following a secondary literature search, i.e., using the search keywords as the specific biomarker(s) in combination with "Leishmaniasis" and/or "Kala-azar", accounting for a total of 89 studies. The biomarkers studied in these patients with VL and PKDL were sub-grouped based on (i) circulatory markers or (ii) nucleic acid markers. Accordingly, for VL and PKDL, nine and six circulatory markers respectively were identified (Tables 1, 4). 


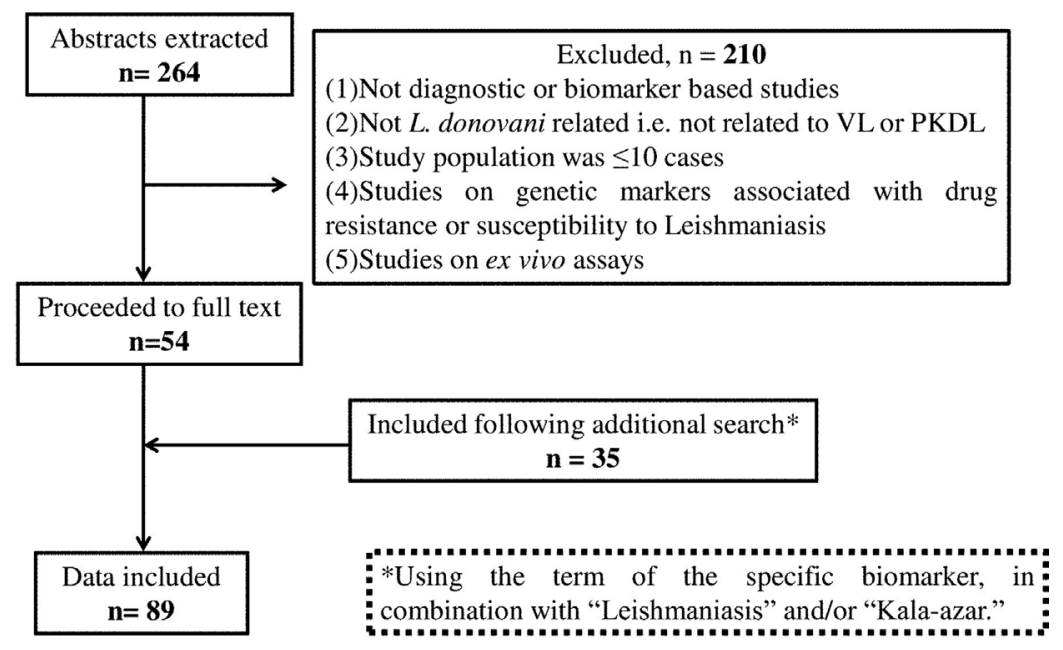

FIGURE 1 | Flow diagram of the studies included.

In case of nucleic acid based markers, eight and five were identified for VL and PKDL, respectively (Tables 2, 5).

\section{Monitoring of VL Circulatory Biomarkers}

In Leishmaniasis, the cytokine microenviroment stimulating polyclonal B cell activation lead to an enhanced isotype switching to IgG1 and IgG3 (Chatterjee et al., 1998; Anam et al., 1999; Bhattacharyya et al., 2014). In addition, soluble lymphokines secreted by T-cells regulate human B cell proliferation and differentiation, along with a predominant Th2 presence that translates into an enhanced presence of anti-leishmanial antibodies (Mukhopadhyay et al., 2012). This has been exploited to develop serological tests for VL with a view to replace invasive procedures for demonstration of parasites in giemsa stained tissue aspirates from spleen, bone marrow, or lymph nodes (Sundar and Rai, 2002).

ELISA based assays using crude or soluble antigens sourced from promastigotes or axenic amastigotes have been used for serodiagnosis of VL, with the rK39-Immunochromatographic test (ICT) being the most robust (Sundar et al., 2006 and references therein; Table 1). Additionally, the heightened antileishmanial IgG and its subclasses have helped identify active VL, and furthermore, reduction of IgG1 titres was associated with successful treatment (Saha et al., 2005; Ansari et al., 2008; Bhattacharyya et al., 2014). However, the ICT/ELISA technique failed to detect HIV/VL co-infected cases, possibly owing to their immunosuppressed status (Medeiros et al., 2017). Another limitation was that the ICT showed positivity in a significant proportion of apparently healthy individuals in endemic regions as also remained positive for long periods after cure from VL (Das et al., 2020). Other Leishmania specific coating antigens are recombinant GP63 (rGP63), recombinant cysteine protease A (rCPA), and soluble leishmania antigen (SLA, Ejazi et al., 2016).

To circumvent the false positivity in patients cured from VL most likely due to the presence of anti-leishmanial antibodies, detection of antigen in urine is an excellent alternative and can be expected to broadly correlate with the parasite load. Till date, target antigens tested by the ELISA method (Table 1) include recombinant GP63 (rGP63), recombinant cysteine protease A (rCPA) and SLA (Ejazi et al., 2016). A major advantage of this approach is its potential to be implemented in a field setting. However, these antigen based tests have shown moderate sensitivity (Table 1), and efforts should therefore be focussed on improving their sensitivity, as also evaluate their potential as a "test of cure".

\section{Nucleic Acid Markers in VL}

Nucleic acid based methods are gaining popularity, especially for monitoring treatment effectiveness, a point very relevant for ensuring success of the Leishmaniasis elimination program. Till date, several molecular based assays such as PCR or quantitative PCR (qPCR) have been validated and serve as reference tools for diagnosis of Leishmaniasis (Salotra et al., 2003; Sundar et al., 2018). The widespread availability of these tests at peripheral health centres to diagnose VL could have a great impact on disease management.

To enable Leishmania detection, a molecular target should have high abundance and this criteria is best achieved by (i) kinetoplast mini-circle DNA (kDNA), present as 1,000 s copies per cell in all Leishmania sp (Salotra et al., 2001; Mary et al., 2004; Mary et al., 2006; Verma et al., 2010) and (ii) 18s rRNA (Deborggraeve et al., 2008; Mehrotra et al., 2011; Srivastava et al., 2011). As a diagnostic tool for VL, the kDNA based qPCR or real-time PCR (Table 2) has stood the test of time (Mary et al., 2006; Verma et al., 2010; Sudarshan et al., 2011; Abbasi et al., 2013; Sudarshan et al., 2015; Hossain et al., 2017). However, its assessment for quantification of relapse or treatment response remain limited (Mary et al., 2006; Verma et al., 2010; Sudarshan et al., 2011; Sudarshan et al., 2015). It is essential that these qPCR approaches be applied to address the last mile challenges of the South Asia Leishmaniasis elimination program (Table 2). 
TABLE 1 | Antigen/Antibody Markers of VL.

\begin{tabular}{|c|c|c|c|c|c|c|c|}
\hline Methods & Target & Biological source & Pre T/tAbsorbances/titres & $\begin{array}{c}\text { Post T/ } \\
\text { tAbsorbances/titres }\end{array}$ & $\begin{array}{l}\text { Sensitivity } \\
\text { (\%) }\end{array}$ & $\begin{array}{l}\text { Specificity } \\
\text { (\%) }\end{array}$ & Reference \\
\hline \multirow[t]{8}{*}{ Latex agglutination (KAtex) } & Carbohydrate antigen & Urine, $n=382$ (Pre); $n=273$ (Post) & 332/382 (86.9\%) & 8/273: (3\%) & 87 & 99 & Sundar et al., 2006 \\
\hline & & Urine, $n=230$ & $169 / 230(73.5 \%)$ & ND & 73.5 & 94 & Sundar et al., 2007 \\
\hline & & Urine, $n=36$ & $27 / 36(75 \%)$ & ND & 75 & 100 & Salam et al., 2011 \\
\hline & & Urine, $n=49$ & $28 / 49$ (57.1\%) & ND & $41.7-73.4$ & 84.3 & Diro et al., 2007 \\
\hline & & Urine, $n=35$ & $18 / 35(51.4 \%)$ & ND & 51.4 & 98.3 & Ben-Abid et al., 2017 \\
\hline & & Oral fluid, $n=35$ & $28 / 35(80 \%)$ & ND & 80 & 88.3 & -do- \\
\hline & A2 amastigote antigen (A2 LAT) & Blood, $n=43$ & $38 / 43(88 \%)$ & ND & 88 & 93.5 & Akhoundi et al., 2013 \\
\hline & Promastigote antigen (Pro LAT) & -do- & $38 / 43(88 \%)$ & ND & 88 & 91 & -do- \\
\hline RBC ELISA & 9-O-acetylated sialic acids & Blood, $n=56$ & $54 / 56(96.4 \%)$ & ND & 96 & 79 & Chava et al., 2002 \\
\hline \multirow[t]{4}{*}{ PEG ELISA } & Leishmania promastigote & Blood, $n=24$ (as confirmed by rk39) & IgG: 23/24: $1.73 \pm 0.11$ & ND & $\lg G=96$ & $\lg G=96.4$ & Datta et al., 2015 \\
\hline & (IgG and IgG subclasses & -do- & $\lg G 1: 12 / 24: 0.32 \pm 0.05$ & ND & $\lg \mathrm{G} 1=50$ & $\operatorname{lgG} 1=100$ & -do- \\
\hline & containing Immune complex) & -do- & $\operatorname{lgG} 2: 7 / 24: 0.44 \pm 0.06$ & ND & $\lg \mathrm{G} 2=29$ & $\lg G 2=100$ & -do- \\
\hline & & -do- & $\lg G 3: 8 / 24: 0.69 \pm 0.04$ & ND & $\lg \mathrm{G} 3=.33 .3$ & $\lg G 3=.100$ & -do- \\
\hline \multirow{24}{*}{$\begin{array}{l}\text { Enzyme-linked } \\
\text { Immunosorbent Assay } \\
\text { (ELISA) }\end{array}$} & $\begin{array}{l}\text { Leishmania promastigote } \\
\text { (IgG subclasses) }\end{array}$ & $\begin{array}{l}\text { Blood, } n=38 \\
\text { (Adults, } n=24 \& \text { Children, } n=14 \text { ) }\end{array}$ & $\begin{array}{l}\text { IgG: Adults, } 1.58 \pm 0.61 \\
\text { Children: } 1.44 \pm 0.61\end{array}$ & ND & ND & ND & Ansari et al., 2008 \\
\hline & & -do- & $\begin{array}{l}\text { IgG1, Adults: } 0.61 \pm 0.31 \\
\quad \text { Children: } 0.61 \pm 0.25\end{array}$ & ND & ND & ND & -do- \\
\hline & & -do- & $\begin{array}{l}\text { IgG3, Adults: } 0.16 \pm 0.11 \\
\text { Children } 0.28 \pm 0.14\end{array}$ & ND & ND & ND & -do- \\
\hline & & -do- & $\begin{array}{l}\text { IgG4, Adults: } 0.15 \pm 0.06 \\
\text { Children } 0.25 \pm 0.02\end{array}$ & ND & ND & ND & -do- \\
\hline & & Blood, $n=66$ (Pre); $n=49$ (Post) & lgG1: 60/66 (90.9\%) & $\operatorname{lgG} 1: 7 / 49(14 \%)$ & 90 & ND & Bhattacharyya et al., 2014 \\
\hline & & $\begin{array}{l}\text {-do- } \\
n=54 \text { (Relapsed) }\end{array}$ & $\begin{array}{c}\text { IgG3: 50/66 (75.7\%) } \\
\text { NA }\end{array}$ & $\begin{array}{l}\text { IgG3: } 7 / 49 \text { (14\%) } \\
\text { Relapsed VL }\end{array}$ & 75 & ND & -do- \\
\hline & & & & $\operatorname{lgG1}: 45 / 54(83.3 \%)$ & 84.2 & ND & -do- \\
\hline & & & & IgG3:29/54 (53.7\%) & 52.6 & ND & -do- \\
\hline & & $\mathrm{n}=80$ as confirmed by rk39 & IgG1: 72/80 (90\%) & ND & lgG1: 90 & IgG: 77.2 & Freire et al., 2019 \\
\hline & & - do- & $\operatorname{lgG} 3: 60 / 80(75 \%)$ & ND & IgG3: 75 & ND & -do- \\
\hline & & -do- & lgG: 66/80 (82.5\%) & ND & IgG: 77 & ND & -do- \\
\hline & Gp63, EF1 $\alpha$, and cysteine & Blood, $n=54$ (Pre and Post t/t) & Gp63:50/54 (92.5\%) & No significant changes & 92.5 & $60-95.2$ & Didwania et al., 2020 \\
\hline & protease $\mathrm{C}(\mathrm{CPC})$ & -do- & EF1 $\alpha: 52 / 54$ (96.2\%) & found & 96.2 & $10-100$ & -do- \\
\hline & & - do- & CPC: 53/54 (98.1\%) & & 98.1 & $65-100$ & -do- \\
\hline & & Urine, $\mathrm{n}=50$ (Pre and Post t/t) & Gp63:45/50 (90\%) & $\begin{array}{l}\text { Levels declined } \\
\text { sharply at }\end{array}$ & 90 & $88.8-100$ & -do- \\
\hline & & -do- & EF1 $\alpha: 42 / 50$ (84\%) & $\begin{array}{l}6 \text { months post } \\
\text { treatment }\end{array}$ & 84 & $55.5-94.7$ & -do- \\
\hline & & -do- & CPC: $48 / 50(96 \%)$ & & 96 & $94.4-100$ & -do- \\
\hline & rK39 & Blood, $n=285$ & 284/285 (99.9\%) & ND & 96.3-99.6 & 75.0-88.3 & $\begin{array}{l}\text { Machado de Assis et al., } \\
2012\end{array}$ \\
\hline & & Blood, $n=84$ & 72/84 (85.7\%) & ND & 85.7 & 86 & $\begin{array}{l}\text { Hosseini Farash et al., } \\
2020\end{array}$ \\
\hline & BHUP1 & Blood, $n=108$ & 103/108 (95.3\%) & ND & 95 & $96-100$ & Kumar et al., 2012 \\
\hline & $\begin{array}{l}\text { Li-rK39 } \\
\text { (Leishmania infantum rK39) }\end{array}$ & Blood, $n=100$ & $96 / 100$ (96\%) & ND & 96.8 & $93.8 \%$ & Rezaei et al, 2019 \\
\hline & rEnolase & Blood, $n=30$ & $30 / 30(100 \%)$ & ND & $83-100$ & 92.3-99 & Duarte et al., 2017 \\
\hline & rA2 & -do- & 28/30(94\%) & ND & $75.1-99.8$ & $62.4-83.9$ & -do- \\
\hline & SLA & -do- & 15/30(50\%) & ND & $27.2-72.8$ & $92.3-99.6$ & -do- \\
\hline
\end{tabular}




\begin{tabular}{|c|c|c|c|c|c|c|c|}
\hline Methods & Target & Biological source & Pre T/tAbsorbances/titres & $\begin{array}{c}\text { Post T/ } \\
\text { tAbsorbances/titres }\end{array}$ & $\begin{array}{l}\text { Sensitivity } \\
\text { (\%) }\end{array}$ & $\begin{array}{l}\text { Specificity } \\
\text { (\%) }\end{array}$ & Reference \\
\hline & rKDDR & Blood, $n=84$ & 74/84 (88.5\%) & ND & 88.5 & 97.3 & Dhom-Lemos et al., 2019 \\
\hline & rEF1b & Blood, $n=30$ & $30 / 30(100 \%)$ & ND & 100 & 100 & Santos et al., 2019 \\
\hline & & Cut off level rEF1b >0.4135 & & & & & \\
\hline & rk28 & Blood, $n=252$ & 251/252 (99.6\%) & ND & 99.6 & $94.7-100$ & Vaish et al., 2012 \\
\hline & rLiHyS & Blood, $n=23$ & 23/23 (100\%) & ND & $94.7-100$ & $93.8-100$ & Dias et al., 2018 \\
\hline & $\begin{array}{l}\text { 40S ribosomal protein } \mathrm{S} 12 \\
\text { antigen }\end{array}$ & $\begin{array}{l}\text { PBMC, } n=14 \text { confirmed by rk39 } \\
\text { and PCR }\end{array}$ & 9/14 (64.3\%) & ND & 68 & ND & Zhang et al., 2018 \\
\hline & LiHyC and PeptC & Blood, $n=30$ & $\mathrm{LiHyC}=30 / 30(100 \%)$ & ND & 100 & ND & Machado et al., 2020 \\
\hline & & -do- & PeptC = 30/30 (100\%) & ND & 100 & ND & -do- \\
\hline & Li-isd1, Li-txn1, Li-ntf2, Ld- & Urine, $n=24$ & Li-isd1 8/24 & ND & 33.3 & ND & Abeijon et al., 2020 \\
\hline & mao1, Ld-ppi1, and Ld-mad1 & - do- & Li-txn1 11/24 & ND & 45.8 & ND & - do- \\
\hline & & -do- & Li-ntf2 7/24 & ND & 29.1 & ND & -do- \\
\hline & & -do- & Ldmao 10/24 & ND & 41.6 & ND & -do- \\
\hline & & -do- & Ld-ppi1 7/24 & ND & 29.1 & ND & -do- \\
\hline & & -do- & Ld-mad1 0/24 & ND & 0 & ND & -do- \\
\hline & Ld-mao1 and Ld-ppi1 & Urine, $n=45$ & Ld-mao1 20/45 & ND & 44.2 & ND & Abeijon et al., 2019 \\
\hline & & -do- & Ld-ppi1 13/45 & ND & 28.8 & ND & -do- \\
\hline & $\mathrm{C} 1, \mathrm{C} 8$, and $\mathrm{C9}$ & Blood, $n=80$ & $\mathrm{C} 1=53 / 80(66.2 \%)$ & ND & 66.2 & 60 & Fonseca et al., 2014 \\
\hline & & - do- & $\mathrm{C} 8=47 / 80(58.2 \%)$ & ND & 58.2 & 50 & -do- \\
\hline & & - do- & $\mathrm{C9}=54 / 80(67.9 \%)$ & ND & 67.9 & 77.7 & - do- \\
\hline & rHRF, rLiHyD, rLiHyT, rLiHyV, & Blood, $n=30$ & $\mathrm{rHRF}=30 / 30(100 \%)$ & ND & 100 & 96.7 & Portela et al., 2018 \\
\hline & rLiHyp6, rA2, or SLA & -do- & rLiHyD = 30/30 (100\%) & ND & 100 & 96.7 & -do- \\
\hline & & -do- & rLiHyt = 30/30 (100\%) & ND & 100 & 96.7 & -do- \\
\hline & & -do- & rLiHyV = 21/30 (70\%) & ND & 70 & 96.7 & -do- \\
\hline & & -do- & rLiHyp6 = 10/30(33.3\%) & ND & 33.3 & 96.7 & -do- \\
\hline & & -do- & $r A 2=16 / 30(53 \%)$ & ND & 53 & 96.7 & -do- \\
\hline & & - do- & SLA $=14 / 30(46.7 \%)$ & ND & 46.7 & 96.7 & -do- \\
\hline & Neopterin & Blood, $n=96$ (Pre \& Post t/t) & 96/96 (100\%) & $4 / 96(4 \%)$ & 100 & 75 & Kip et al., 2018 \\
\hline & Adenosine Deaminase (ADA) & Blood, Pre, and Post t/t; n = 39 & $\begin{array}{l}\text { Significantly higher than } \\
\text { healthy controls }\end{array}$ & $\begin{array}{l}\text { Significantly reduced } \\
\text { by } 3 \text {-fold }\end{array}$ & ND & ND & $\begin{array}{l}\text { Vijayamahantesh et al., } \\
2016\end{array}$ \\
\hline \multirow{17}{*}{$\begin{array}{l}\text { Immuno chromatographic } \\
\text { test (ICT) }\end{array}$} & rK39 & Blood, $n=150$ & 149/150 (99.3\%) & ND & 99.3 & 89 & Sundar et al., 2006 \\
\hline & & Blood, $n=49$ & 46/49 (93.8\%) & ND & 95.3 & 62.7 & Diro et al., 2007 \\
\hline & & Blood, $n=100$ & 99/100 (99\%) & ND & 99 & 95 & Boelaert et al., 2008 \\
\hline & & Blood, $\mathrm{n}=94$ & 94/94 (100\%) & ND & 100 & 87 & Mandal et al., 2008 \\
\hline & & Blood, $n=100$ (Pre); $n=50$ (post) & 98/100 (98\%) & $25 / 50(50 \%)$ & 98 & 100 & Singh et al., 2009 \\
\hline & & Blood, $n=624$ & 251/624 (40.2\%) & ND & 91 & 93 & Matlashewski et al., 2013 \\
\hline & & Blood, $n=145$ & $105 / 145$ (72.4\%) & ND & 72.4 & 99.6 & Moura et al., 2013 \\
\hline & & Blood, $n=231$ & 204/231 (88.3\%) & ND & 95.8 & 98.7 & Gao et al., 2015 \\
\hline & & Blood, $n=38$ & 25/38 (65.8\%) & VL Relapsed, 10/10 & 67 & 85 & Mondal et al., 2019 \\
\hline & & VL Relapsed, $\mathrm{n}=10$ & & (100\%); T/t Failure, 4/7 & & & \\
\hline & & T/t Failure, $n=7$ & & $(57.1 \%)$ & & & \\
\hline & & Blood, $n=51$ & 49/51 (96.1\%) & ND & 83 & ND & Freire et al., 2019 \\
\hline & & Blood, $n=128$ & 115/128 (89.8\%) & ND & 83-94 & $90.5-98.8$ & Sanchez et al., 2020 \\
\hline & & -do- & 116/128 (90.6\%) & ND & $84.3-94.6$ & $96.5-100.0$ & -do- \\
\hline & & Saliva, $n=128$ & $94 / 128(73.4 \%)$ & ND & $65-80.3$ & $89.5-98.0$ & -do- \\
\hline & & Blood, $n=98$ & 73/98 (74.5\%) & ND & 80 & 82 & Chappuis et al., 2003 \\
\hline & & Blood, $n=200$ & $189 / 200(94.5 \%)$ & ND & $88.0-95.4$ & $95.7-100$ & Mukhtar et al., 2015 \\
\hline
\end{tabular}




\begin{tabular}{|c|c|c|c|c|c|c|c|}
\hline Methods & Target & Biological source & Pre T/tAbsorbances/titres & $\begin{array}{c}\text { Post T/ } \\
\text { tAbsorbances/titres }\end{array}$ & $\begin{array}{l}\text { Sensitivity } \\
(\%)\end{array}$ & $\begin{array}{l}\text { Specificity } \\
(\%)\end{array}$ & Reference \\
\hline & rk28 & Urine, $n=87$ & $82 / 87(94.3 \%)$ & ND & 94.3 & $87.1-97.2$ & Ghosh et al., 2016 \\
\hline & & Blood, $n=87$ & $85 / 87(97.7)$ & ND & 97.7 & $91.9-97.2$ & - do- \\
\hline & & Blood, $n=219$ & $186 / 219$ (84.9\%) & ND & 77 & 96 & da Silva et al., 2018 \\
\hline & rKE16 & Blood, $n=131$ & $104 / 131(79 \%)$ & ND & ND & ND & Mbui et al., 2013 \\
\hline & & Blood, $n=200$ & 191/200 (95.5\%) & ND & $92-98$ & $91-100$ & Vaish et al., 2012 \\
\hline & rK39 (lgG/lgM) & Blood, $\mathrm{n}=186$ & $107 / 186(57.5 \%)$ & ND & 91.2 & 95.3 & Freire et al., 2018 \\
\hline \multirow[t]{8}{*}{ DAT } & Parasite promastigote & Blood, $n=184$ & $\begin{array}{l}\text { cut-off titre (1:400) } \\
139 / 184(75.5 \%)\end{array}$ & ND & 97 & ND & Chappuis et al., 2003 \\
\hline & & Blood, $n=150$ & $\begin{array}{c}\geq 1: 1,600 \\
147 / 150(98 \%)\end{array}$ & ND & 96 & 96.5 & Singh et al., 2005 \\
\hline & & Blood, $n=15$ & $\geq 1: 1,600 ; 12 / 15$ (80\%) & ND & $94-97$ & 85 & Adams et al., 2012 \\
\hline & & - do- & $\geq 1: 3,200: 11 / 15$ (73.3\%) & ND & 99 & 100 & - do- \\
\hline & & Blood, $n=110$ & $\geq 1: 800 ; 109 / 110$ (99.1\%) & ND & 60 & ND & Oliveira et al., 2013 \\
\hline & & Blood, $n=405$ & $\begin{array}{l}\text { No cut off stated: } 246 / 405 \\
(60.7 \%)\end{array}$ & ND & $93-95.2$ & 97.5 & Bangert et al., 2018 \\
\hline & & Blood, $n=49$ & $\geq 1: 3,200 ; 46 / 49$ (93.8\%) & ND & 97 & $62-65.3$ & Diro et al., 2007 \\
\hline & & Blood, $n=285$ & 252/285 (88.4\%) & ND & $84.1-92.0$ & $89.2-98.1$ & $\begin{array}{l}\text { Machado de Assis et al., } \\
2012\end{array}$ \\
\hline \multirow[t]{5}{*}{ Dipstick test } & LAg & Blood, $n=463$ & 449/463 (96.9\%) & ND & ND & ND & Ejazi et al., 2019 \\
\hline & $\mathrm{LAg}$ & Urine, $n=97$ & 93/97 (95.8\%) & ND & 95.8 & 100 & Ejazi et al., 2016 \\
\hline & rGP63 & & ND & & & & do \\
\hline & rCPA & & ND & & & & do \\
\hline & SLA & & ND & & & & do \\
\hline Lateral Flow Device & $\beta$-tubulin and LiHyp1 & Blood, $n=24$ & 20/24 (83.3\%) & ND & 90 & $96-100$ & Humbert et al., 2019 \\
\hline \multirow[t]{3}{*}{ IFAT } & $\lg G$ & Blood, $n=405$ & $114 / 405(28.1 \%)$ & ND & 79.4 & 99 & Bangert et al., 2018 \\
\hline & & Blood, $n=104$ & 103/104 (99\%) & ND & 100 & 99 & Cañavate et al., 2011 \\
\hline & & Blood, $n=285$ & 251/285 (88.5\%) & ND & 84.0-92.0 & $75.0-88.2$ & $\begin{array}{l}\text { Machado de Assis et al., } \\
2012\end{array}$ \\
\hline
\end{tabular}




\begin{tabular}{|c|c|c|c|c|c|c|c|}
\hline Methods & Target & Biological material & $\begin{array}{c}\begin{array}{c}\text { Parasite load Pre T/t (\% } \\
+ \text { +ve) }\end{array}\end{array}$ & Parasite load Post $t / t$ & $\begin{array}{l}\text { Sensitivity } \\
\text { (\%) }\end{array}$ & $\begin{array}{l}\text { Specificity } \\
(\%)\end{array}$ & References \\
\hline \multirow{8}{*}{$\begin{array}{l}\text { PCR followed by Southern } \\
\text { Blotting } \\
\text { qPCR }\end{array}$} & Leishmania kDNA & Blood $(n=51)$ & $n=49 / 51(96 \%)$ & ND & 96 & 96 & Salotra et al., 2001 \\
\hline & Leishmania kDNA & Blood, $n=51$ & *837 parasites/ml & *LAmB, 1 parasite/ml (day 60) & 96.5 & 100 & Mary et al., 2004 \\
\hline & & Blood, $n=147$ & ${ }^{\star} 70,560$ parasites $/ \mathrm{ml}$ & *LAmB, 1 parasite/ml (day 5) & 95 & 100 & Mary et al., 2006; \\
\hline & & Blood, $n=31$ & ${ }^{\star} 8,372$ parasites $/ \mathrm{ml}$ & $\begin{array}{c}\text { *AmphoB, } 1 \text { parasite/ml (day 30) } \\
{ }^{*} \text { `SAG: } 290 \text { parasites } / \mathrm{ml} \text { (day 28) } \\
\text { *LAmB, } 72 \text { parasite genomes } / \mathrm{ml} \\
\text { (day } 7 \text { ) }\end{array}$ & ND & ND & Verma et al., 2010 \\
\hline & & Blood, $n=46$ & *894 parasite genomes/ml & *8 parasite genomes/ml (day 30) & 100 & ND & Sudarshan et al., 2011 \\
\hline & & Blood, $n=40$ & $\begin{array}{l}{ }^{*} 574.5 \text { parasites } \\
\text { genomes } / \mathrm{ml}\end{array}$ & ND & 100 & ND & Sudarshan et al., 2015 \\
\hline & & Blood, $n=40$ & *192.69 parasites/ml & $\begin{array}{c}{ }^{*} \text { LAmB, } 0.1 \text { parasites } / \mathrm{ml} \text { (days } \\
\text { not stated) }\end{array}$ & 100 & ND & Hossain et al, 2017 \\
\hline & & Blood, $n=59$ & $\begin{array}{c}\mathrm{n}=13 / 59(22 \%) \\
1-10 \text { parasites } / \mathrm{ml} \\
\mathrm{n}=23 / 59(39 \%) \\
10-100 \text { parasites/ml } \\
\mathrm{n}=19 / 59(32 \%) \\
1,000 \text { and above } \\
\text { parasites } / \mathrm{ml}\end{array}$ & ND & ND & ND & Abbasi et al., 2013 \\
\hline \multirow[t]{12}{*}{ PCR } & 18s rRNA & Blood, $n=140$ & $129 / 140(92.1 \%)$ & ND & 92.1 & 99.64 & Deborggraeve et al., 2008 \\
\hline & & Bone Marrow, $n=170$ & 158/170 (92.9\%) & ND & 92.9 & 94.60 & \\
\hline & & Blood, $n=500$ & $439 / 500$ (87.8\%) & ND & 87.8 & 99.64 & Srivastava et al., 2011 \\
\hline & & Buccal swab, $n=148$ & $123 / 148(83.1 \%)$ & ND & 83 & 90.56 & Mehrotra et al., 2011 \\
\hline & OligoC- Test & Blood, $n=84$ & $81 / 84(96.4 \%)$ & ND & 96.4 & 88.8 & Basiye et al., 2010 \\
\hline & & Blood, $n=79$ & $76 / 79(96.2 \%)$ & ND & 96.2 & 90 & Saad et al., 2010 \\
\hline & & Splenic Aspirate, $\mathrm{n}=31$ & $30 / 31$ (96.8\%) & ND & 96.8 & 90 & -do- \\
\hline & & Bone Marrow, $n=64$ & 62/64 (96.9\%) & ND & 96.9 & 90 & - do- \\
\hline & Nucleic acid sequence-based & Blood, $n=84$ & 67/84 (79.8\%) & ND & 79.8 & 100 & Basiye et al., 2010 \\
\hline & amplification oligochromatography & Blood, $n=79$ & $76 / 79$ (96.2\%) & ND & 96.2 & 100 & Saad et al., 2010 \\
\hline & & Splenic Aspirate, $n=31$ & $30 / 31$ (96.8\%) & ND & 96.8 & 100 & -do- \\
\hline & (NASBA-OC) & Bone Marrow, $n=64$ & $61 / 64(96.4 \%)$ & ND & 95.3 & 100 & -do- \\
\hline Loop Mediated Isothermal & Leishmania kDNA and 18s rRNA & Splenic aspirate, $n=75$ & $68 / 75(90.7 \%)$ & ND & 90.7 & 100 & Khan et al., 2012 \\
\hline Amplification & & Splenic aspirate, $\mathrm{n}=15$ & $53 / 55(96.3 \%)$ & ND & 100 & 98.5 & Verma et al., 2013a \\
\hline \multirow[t]{7}{*}{ LAMP } & & Buffy Coat, $n=84$ & 80/84 (95.2\%) & ND & 97.65 & 99.01 & Mukhtar et al., 2018 \\
\hline & & Buffy Coat, $n=50$ & $46 / 50(92 \%$ & ND & 92 & 100 & Adams et al., 2018 \\
\hline & & Blood, $n=55$ & $53 / 55$ (96.4\%) & ND & 96.4 & 98.5 & Verma et al., 2013a \\
\hline & & Blood, $n=47$ & $44 / 47$ (93.6\%) & ND & 93.6 & 100 & Ghasemian et al., 2014 \\
\hline & & Blood, $n=38$ & $35 / 38$ (92.1\%) & ND & 97 & 100 & Abbasi et al., 2016 \\
\hline & & Blood, $\mathrm{n}=66$ & $64 / 66(96.9 \%)$ & ND & 96.9 & 100 & Verma et al., 2017 \\
\hline & & Blood, $\mathrm{n}=179$ & $176 / 179(98.3 \%)$ & ND & 98.32 & 96.59 & Dixit et al., 2018 \\
\hline $\begin{array}{l}\text { Direct Blood Lysis- } \\
\text { LAMP }\end{array}$ & Leishmania kDNA and 18s rRNA & Blood, $n=72$ & 67/72 (93\%) & ND & $93.06 \%$ & 100 & Dixit et al., 2018 \\
\hline $\begin{array}{l}\text { Recombinase Polymerase } \\
\text { Amplification Assay }\end{array}$ & Leishmania kDNA & Blood, $n=23$ & 23/23 (100\%) & ND & 100 & ND & Mondal et al., 2016 \\
\hline \multirow{2}{*}{ Gene sequencing } & Leishmania kDNA & Bone marrow, $n=22$ & 20/22 (90.9\%) & ND & 91 & 100 & Hu et al., 2000 \\
\hline & & Blood, $n=16$ & $11 / 16(68.7 \%)$ & ND & 69 & 100 & - do- \\
\hline $\begin{array}{l}\text { PCR-High resolution } \\
\text { melting HRM }\end{array}$ & Leishmania kDNA & Bone Marrow, $n=30$ & 28/30 (90.9\%) & ND & ND & ND & Pita-Pereira et al., 2012 \\
\hline
\end{tabular}


Taken together, it is reasonable to propose that PCR methods could perhaps replace the conventional microscopic detection of LD bodies in giemsa stained tissue aspirates for monitoring of VL, the latter approach being fraught with limitations that include invasiveness and low sensitivity, especially post treatment. However, molecular tests carry the burden of cost, time, necessity for specialized personnel and equipment, as also a stable cold chain to minimize chances of denaturation of reagents and samples. It can be envisaged that following the COVID-19 pandemic, upgrading of the government funded molecular diagnostic facilities could potentially be harnessed for control programs pertaining to Neglected Tropical Diseases such as VL and PKDL.

An emerging option translatable to a field scenario is the Loop-mediated isothermal amplification (LAMP) assay (Table 2), which includes a quick and easy DNA extraction method, such as "boil and spin" (Notomi et al., 2000). However, the main issue with LAMP is false positivity as also the assay requires the use of six primers which increases the possibility of a primer-dimer formation. Bst DNA polymerase is commonly used in LAMP because they have strong strand displacement activity (required for isothermal techniques), but being unstable $>70^{\circ} \mathrm{C}$, it cannot be used in conventional PCR where the denaturation step is close to $90^{\circ} \mathrm{C}$ (Vink et al., 2018; Nzelu et al., 2019). Considering its field applicability, studies pertaining to the efficacy of LAMP assay in monitoring treatment should be undertaken.

Another promising approach is the recombinase polymerase amplification (RPA) assay used for detection of VL, developed in the format of a mobile suitcase laboratory and could be effective in a resource limited setting (Table 2). However, the test needs validation on a larger sample pool, as also its ability to monitor treatment outcome needs to be evaluated.

\section{Circulatory and Nucleic Acid Biomarkers in HIV-VL Co-Infection}

The detection of anti-leishmanial antibodies in HIV-VL co-infected patients is challenging as the associate immunological dysfunction accounts for the lowered sensitivity of serological tests (Cota et al., 2012). In resource limited settings, DAT and immunoblotting as compared to ELISA and the immunofluorescence antibody test have shown moderate sensitivity ( 81 and $84 \%$, respectively, Salotra et al., 1999; ter Horst et al., 2009, Table 3). The low antibody titres could be augmented using recombinant polypeptides, but requires

TABLE 3 | Antibody and Nucleic Acid Markers of HIV-VL.

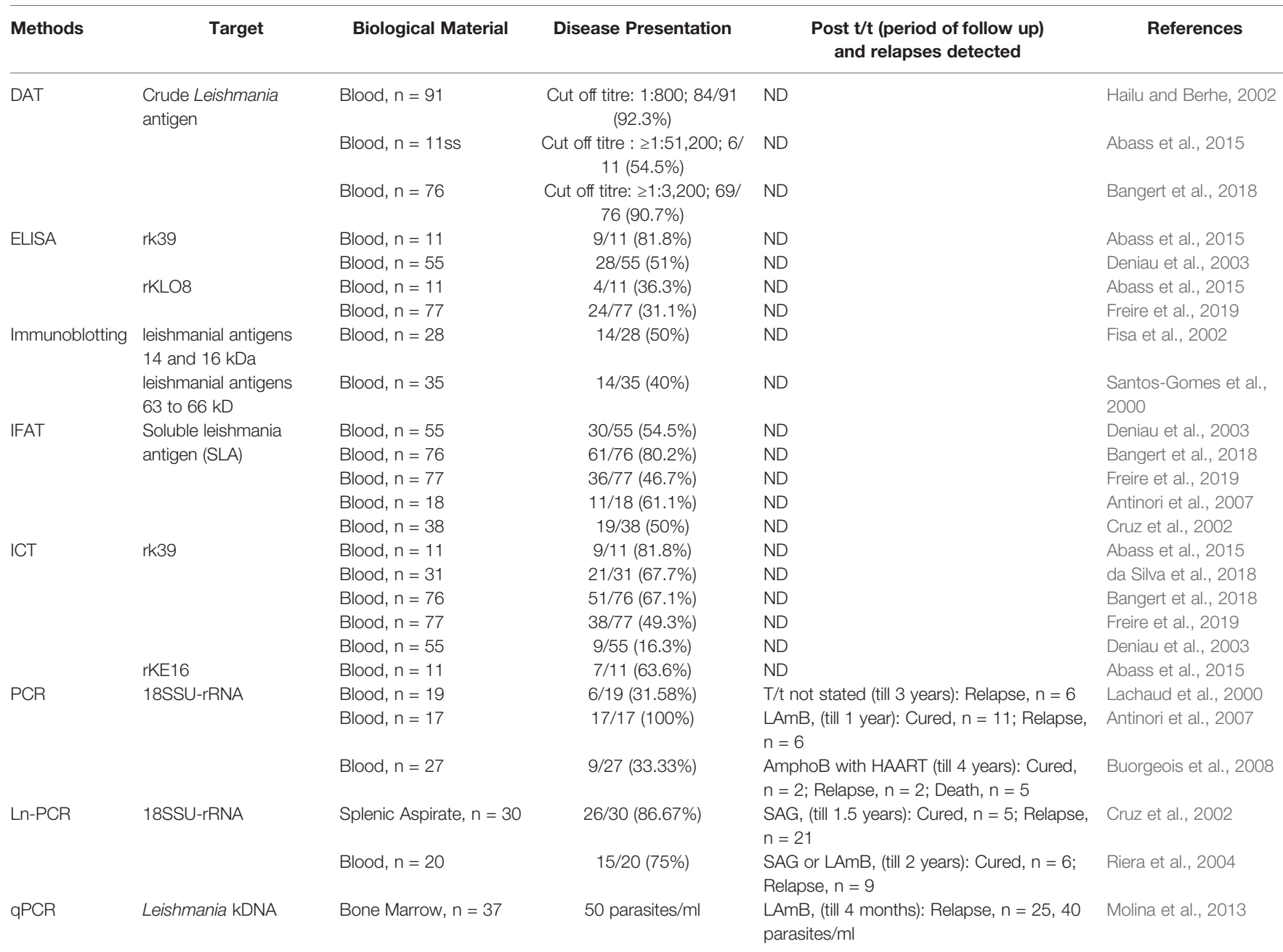


TABLE 4 | Antigen/Antibody Markers of PKDL.

\begin{tabular}{|c|c|c|c|c|c|c|c|c|}
\hline Methods & Target & $\begin{array}{l}\text { Biological } \\
\text { source }\end{array}$ & PKDL Variants & Pre $t / t$ & Post $\mathrm{t} / \mathrm{t}$ & $\begin{array}{l}\text { Sensitivity } \\
(\%)\end{array}$ & $\begin{array}{l}\text { Specificity } \\
(\%)\end{array}$ & Reference \\
\hline \multirow[t]{7}{*}{ ICT } & \multirow[t]{7}{*}{ rk39 } & Blood & All types, $n=50$ & $50 / 50(100 \%)$ & ND & 100 & 100 & Verma et al., 2013a \\
\hline & & -do- & Polymorphic, $n=74$ & $70 / 74(91 \%)$ & ND & 91 & 100 & Salotra et al., 2003 \\
\hline & & & Macular, $n=14$ & $11 / 14(78.6 \%)$ & ND & & & \\
\hline & & -do- & All types, $n=32$ & 30/32 (93.7\%) & ND & 67 & 85 & Mondal et al., 2019 \\
\hline & & -do- & All types, $n=6$ & 6/6 (100\%) & ND & 100 & 100 & Mathur et al., 2006 \\
\hline & & -do- & All, $n=35$ & $35 / 35(100 \%)$ & ND & 100 & 100 & Topno et al., 2018 \\
\hline & & -do- & $\begin{array}{l}\text { Polymorphic, } n=20 \\
\text { Macular, } n=5\end{array}$ & $\begin{array}{l}19 / 20(95 \%) \\
5 / 5(100 \%)\end{array}$ & ND & 96 & 100 & Das et al., 2007 \\
\hline \multirow[t]{8}{*}{ DAT } & \multirow[t]{8}{*}{ Leishmania Promastigote } & Blood & Polymorphic, $n=46$ & $\leq 1: 6,400$ & ND & 1:400: 98.5 & 1:400: 84.2 & Singh et al., 2005 \\
\hline & & -do- & Macular, $\mathrm{n}=22$ & Poly: 40/46 (86.9\%) & & 1:800: 98.5 & 1:800: 96 & -do- \\
\hline & & & & Mac: 13/22 (59.1\%) & & $\begin{array}{l}\text { 1:1,600: } \\
94.2\end{array}$ & 1:1,600: 100 & -do- \\
\hline & & & & & & $\begin{array}{l}1: 3,200: \\
77.2\end{array}$ & 1:3,200: 100 & -do- \\
\hline & & & Polymorphic, n = 20 & 1:800-1:12,800 & ND & ND & ND & Verma et al., 2015 \\
\hline & & & Macular, $n=25$ & Poly: 19/20 (95\%) & & & & \\
\hline & & & papuloerythematous & Mac: $21 / 25(84 \%)$ & & & & \\
\hline & & & $n=15$ & $\begin{array}{c}\text { Papuloerythematous: 14/15 } \\
(93.3 \%)\end{array}$ & & & & \\
\hline \multirow{8}{*}{$\begin{array}{l}\text { Subclass ELISA } \\
\text { (IgG, lgG1, IgG2, } \\
\text { and lgG3) }\end{array}$} & \multirow[t]{8}{*}{ Leishmania Promastigote } & \multirow[t]{4}{*}{ Blood } & Polymorphic, $n=20$ & $\begin{array}{c}\text { IgG: } 23 / 23(100 \%), \text { cut off } \mathrm{OD}= \\
0.27\end{array}$ & ND & $\lg G=100$ & $\lg G=96$ & Saha et al., 2005 \\
\hline & & & Macular, $\mathrm{n}=3$ & $\begin{array}{c}\text { IgG1: } 21 / 23(91.3 \%), \text { cut off OD } \\
=0.48\end{array}$ & & $\lg G 1=91$ & $\operatorname{lgG} 1=96.7$ & -do- \\
\hline & & & & $\begin{array}{c}\text { IgG2: } \begin{aligned} 10 / 23 & (43.5 \%), \text { cut off OD } \\
& =0.45\end{aligned}\end{array}$ & & $\lg G 2=45.5$ & $\operatorname{lgG} 2=93.3$ & -do- \\
\hline & & & & $\begin{array}{c}\text { IgG3: } 19 / 23 \text { (82.6), cut off } \mathrm{OD}= \\
0.25\end{array}$ & & $\lg G 3=81.8$ & $\lg G 3=83$ & -do- \\
\hline & & \multirow[t]{4}{*}{ Blood } & \multirow[t]{2}{*}{$\begin{array}{l}\text { Polymorphic, } n=41 \\
n=22(\text { end of } t / t)\end{array}$} & $\begin{array}{l}\text { IgG: Poly: } 0.62 \text { [0.27-0.92], } \\
\text { Mac: } 0.36 \text { [0.17-0.56], }\end{array}$ & Poly : IgG1 and lgG3 levels & ND & ND & $\begin{array}{l}\text { Mukhopadhay } \\
\text { et al., } 2012\end{array}$ \\
\hline & & & & $\begin{array}{l}\text { IgG1: Poly: } 0.41[0.26-0.87] \\
\text { Mac: } 0.33[0.12-0.41]\end{array}$ & $\begin{array}{c}\text { decreased significantly }(p< \\
0.01 \text { and }\end{array}$ & ND & ND & -do- \\
\hline & & & \multirow[t]{2}{*}{$\begin{array}{l}\text { Macular, } \mathrm{n}=16 \mathrm{pr} \\
\mathrm{n}=6 \text { post (end of } \mathrm{t} / \mathrm{t})\end{array}$} & $\begin{array}{l}\text { IgG2: Poly: } 00.08[0.00-0.16] \\
\text { Mac: } 0.11[0.04-0.22]\end{array}$ & $p<0.001$ & ND & ND & -do- \\
\hline & & & & $\begin{array}{l}\text { IgG3: Poly: } 0.55 \text { [0.20-0.61] } \\
\text { Mac: ND }\end{array}$ & $\begin{array}{l}\text { Mac: neither lgG1 nor lgG3 } \\
\text { levels decreased }\end{array}$ & ND & ND & -do- \\
\hline
\end{tabular}




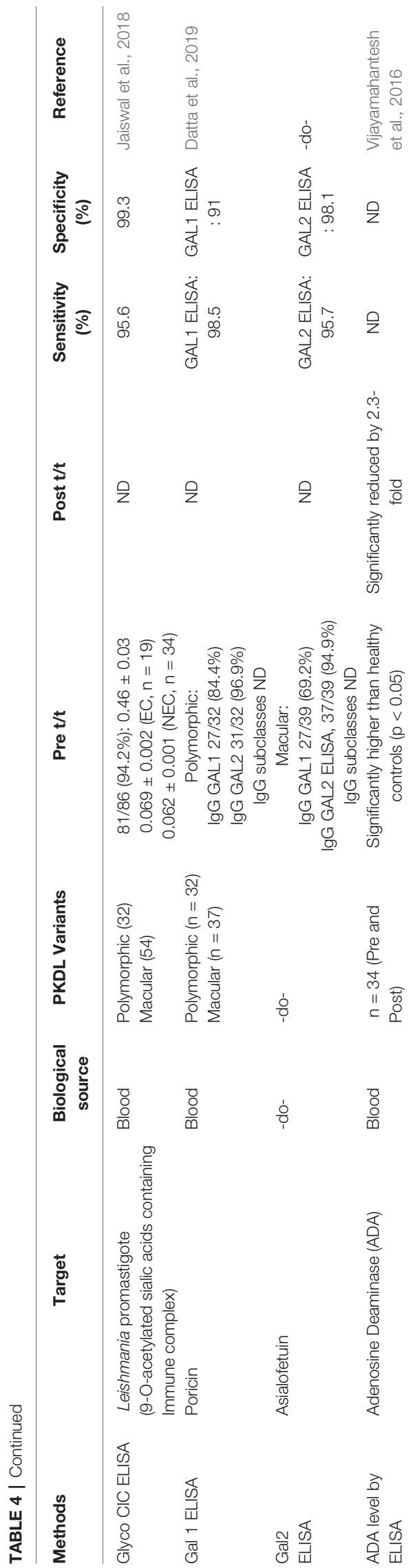

optimization (Lindoso et al., 2018). In studies where confirmation of VL infection was done by microscopy, the antibody tests showed varied sensitivity (Mathur et al., 2006; Redhu et al., 2006; Sinha et al., 2006), emphasizing the need for molecular based assays. Till date, PCR has often been applied as the primary method of detection (Cota et al., 2012), and amplification of 18s rRNA and kDNA regions have proven to be the best option. Real-time PCR has been adopted for diagnosis of HIV-VL co-infected patients (Table 3), where a high parasite burden was reported, but the study population was relatively small (Molina et al., 2013). Further studies are required to monitor the treatment efficacy of these HIV-VL cases.

\section{Monitoring of PKDL Clinical Biomarkers}

As the confirmatory diagnosis of PKDL requires a skin biopsy, it is often not performed, and decisions are made based on clinical assessment and a past history of VL (Ganguly et al., 2010). Based on the lesion types, cases with hypopigmented macules are considered as macular PKDL, whereas cases with an assortment of papules, nodules, macules, and/or plaques are termed as polymorphic PKDL (Zijlstra et al., 2003). Irrespective of the geographic region, the differential diagnosis of $\mathrm{PKDL}$ includes leprosy, vitiligo, pitryasis alba, and miliaria rubra (Zijlstra, 2019). This can lead to misdiagnosis especially in cases where there is no previous history of VL (Das et al., 2011; El Hassan et al., 2013; Ramesh et al., 2015a). In Africa (mainly Sudan), maculopapular rashes are commonest (90\% of cases) and in advanced cases, the papules coalesce to form nodules or plaques that can be confused with leprosy, vitiligo, pityriasis versicolor, tinea corporis, tinea barbae, pityriasis alba (Zijlstra, 2019). Presently, a major challenge is the inability to detect LD bodies in macular cases, which in recent studies have been shown to constitute a substantial component of the burden of PKDL (Zijlstra et al., 2017; Sengupta et al., 2019). Furthermore, in macular cases as hypopigmentation persists even after parasite clearance, it endorses the need for developing an objective and quantifiable "test of cure".

\section{Circulatory Biomarkers}

Among the serological tests for PKDL, the rK39 ICT is the most sensitive, rapid, field applicable, and cost-effective tool. However, it is not completely reliable, since a positive ICT could be attributed to a past episode of VL. Similarly, DAT has also been found to be applicable in field conditions, but shares the same disadvantage as ICT (Table 4). Another challenge is the microscopic detection of parasites in PKDL lesions (Singh et al., 2015) especially the macular variant (Mondal et al., 2010; Nasreen et al., 2012; Verma et al., 2013a; Bhargava et al., 2018; Ghosh et al., 2018).

\section{Nucleic Acid Biomarkers}

A confirmed diagnosis of PKDL is necessary for clinical trials, and is currently based on the detection of Leishmania parasites by microscopy in a slit skin smear. Generally, parasites are present in most papulonodular lesions but in macular lesions, the positivity rate can be as low as $20-40 \%$ (Ramesh et al., 2015a; 
TABLE 5 | Nucleic Acid Markers of PKDL.

\begin{tabular}{|c|c|c|c|c|c|c|c|c|}
\hline Methods & Target & $\begin{array}{l}\text { Tissue } \\
\text { source }\end{array}$ & PKDL variant & $\begin{array}{l}\text { +ve at disease presentation,par- } \\
\text { asite load, }(\%+\mathrm{ve})\end{array}$ & Parasite loadPost $T / t$ & $\begin{array}{l}\text { Sensitivity } \\
\text { (\%) }\end{array}$ & $\begin{array}{l}\text { Specificity } \\
(\%)\end{array}$ & Reference \\
\hline \multirow[t]{2}{*}{ PCR } & kDNA & Skin biopsy & $n=48$ & $45 / 48$ & ND & 93.8 & 100 & Salotra et al., 2001 \\
\hline & & Skin biopsy & $\mathrm{n}=25$ & $24 / 25$ & ND & 96.0 & 100 & Salotra et al., 2003 \\
\hline PCR & 18s rRNA & Skin biopsy & $n=25$ & $24 / 25$ & ND & ND & ND & Srivastava et al., 2011 \\
\hline \multirow[t]{11}{*}{ qPCR } & Leishmania $k D N A$ & Skin biopsy & $\begin{array}{l}\text { Macular, } \mathrm{n}=2 \\
\text { Indurated, } \mathrm{n}=11 \\
\text { Polymorphic, } \mathrm{n}=13\end{array}$ & $\begin{array}{l}\text { Pooled, } \mathrm{n}=26 \\
667(3-240,000) / \mu \mathrm{g} \text { gDNA }\end{array}$ & $\begin{array}{l}\text { Miltefosine (Day 84) } \\
; \text { Pooled, } \mathrm{n}=15 \\
\text { All were -ve }\end{array}$ & 100 & ND & Ramesh et al., 2011 \\
\hline & & Slit aspirate & $\begin{array}{l}\text { Nodular, } \mathrm{n}=26 \\
\text { Papular/Macular, } \mathrm{n}=24\end{array}$ & $\begin{array}{l}9,790 \text { parasites } / \mu \mathrm{l} \text { slit aspirate } \\
427 \text { parasites } / \mu \mathrm{l} \text { slit aspirate }\end{array}$ & $\begin{array}{l}\text { SAG or Miltefosine (Day 30); Pooled, } \\
\mathrm{n}=19\end{array}$ & 100 & 100 & Verma et al., 2013a \\
\hline & & Skin Biopsy & $\begin{array}{l}\text { Nodular, } \mathrm{n}=26 \\
\text { Papular/Macular, } \mathrm{n}=20\end{array}$ & $\begin{array}{l}38,205 \text { parasites } / \mu \mathrm{g} \text { of tissue DNA } \\
599 \text { parasites } / \mu \mathrm{g} \text { of tissue DNA }\end{array}$ & $17 / 19(89.5 \%)$ were negative & ND & ND & -do- \\
\hline & & Slit aspirate & $\begin{array}{l}\text { Polymorphic, } n=59 \\
\text { Macular, } n=14 \\
1 \text { month post } t / t\end{array}$ & 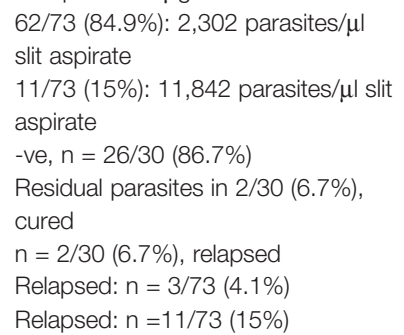 & $\begin{array}{l}<10 \text { parasites } / \mu \text { slit aspirate } \\
<10 \text { parasites } / \mu \text { s slit aspirate }\end{array}$ & ND & ND & Ramesh et al., 2015b \\
\hline & & Slit Aspirate & $\begin{array}{l}\text { Macular, } \mathrm{n}=4 \\
\text { Papular, } \mathrm{n}=20 \\
\text { Nodular, } \mathrm{n}=26\end{array}$ & 60 parasites $/ \mu \mid$ & ND & 77 & ND & Bhargava et al., 2018 \\
\hline & & Skin Biopsy & $\begin{array}{l}\text { Macular, } \mathrm{n}=4 \\
\text { Papular, } \mathrm{n}=17 \\
\text { Nodular, } \mathrm{n}=26\end{array}$ & 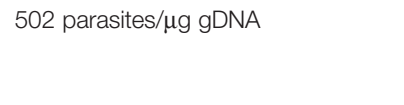 & ND & ND & ND & -do- \\
\hline & & Skin biopsy & $\begin{array}{l}\text { Macular, } n=91 \\
\text { Polymorphic, } n=93\end{array}$ & $\begin{array}{l}3,665(615-21,528) / \mu \mathrm{g} \text { gDNA } \\
18,620(1,266-93,934) / \mu \mathrm{g} \text { gDNA }\end{array}$ & $\begin{array}{l}\text { Miltefosine (day } 84) \text { : } \\
\text { Macular, } n=17,<10 / \mu \mathrm{g} \text { gDNA } \\
\text { Polymorphic, } \mathrm{n}=21,<10 / \mu \mathrm{g} \text { gDNA } \\
\text { LAmB (day } 21) \\
2,128(544-5,763) / \mu \mathrm{g} \text { gDNA } \\
2,541(650-9,073) / \mu \mathrm{g} \text { gDA }\end{array}$ & ND & ND & Moulik et al., 2018 \\
\hline & Leishmania REPL & Skin biopsy & All types, $n=20$ & $20 / 20(100 \%)$ & ND & 100 & 100 & Mondal et al., 2016 \\
\hline & $\begin{array}{l}\text { repeats } \\
(\text { L42486.1) }\end{array}$ & Skin biopsy & $\begin{array}{l}\text { Macular, } \mathrm{n}=38 \\
\text { Papular, } \mathrm{n}=2\end{array}$ & $\begin{array}{l}34 / 40(85 \%) \\
295.46(1.38-4,065.89) \text { parasites/ug } \\
\text { gDNA }\end{array}$ & $\begin{array}{l}\text { Miltefosine (day 84) } \\
\text { qPCR: } \mathrm{n}=3+\mathrm{ve}\end{array}$ & 85 & 100 & Hossain et al., 2017 \\
\hline & & Skin biopsy & Macular, $n=91$ & $83 / 91(91.21 \%)$ & ND & 50.6 & 100 & Ghosh et al., 2018 \\
\hline & & Blood & Macular, $\mathrm{n}=91$ & Negative $(n=91)$ & ND & 91.2 & 100 & \\
\hline \multirow[t]{4}{*}{ Ln-PCR } & SSU-rRNARegion & $\begin{array}{l}\text { Blood, buffy } \\
\text { coat }\end{array}$ & Macular, $n=28$ & $14 / 28(50 \%)$ & ND & ND & ND & Mondal et al., 2010 \\
\hline & Leishmania kDNA & Skin biopsy & $\begin{array}{l}\text { Popymorphic, } n=21 \\
\text { Macular, } n=8\end{array}$ & $27 / 29$ & ND & 93 & ND & Sreenivas et al., 2004 \\
\hline & & Skin biopsy & $\begin{array}{l}\text { Macular, } \mathrm{n}=74 \\
\text { Papular, } \mathrm{n}=33 \\
\text { Nodular, } \mathrm{n}=3\end{array}$ & $\begin{array}{l}\text { 69/74 (93.2\%) } \\
32 / 33(97 \%) \\
3 / 3(100 \%)\end{array}$ & $\begin{array}{l}N D \\
N D \\
N D\end{array}$ & 94.5 & ND & Nasreen et al., 2012 \\
\hline & & Skin biopsy & $\begin{array}{l}\text { Macular, } \mathrm{n}=38 \\
\text { Papular, } \mathrm{n}=2\end{array}$ & $21 / 40(52.5 \%)$ & $\begin{array}{l}\text { Miltefosine (day } 84 \text { ) } \\
\text { Ln-PCR: } n=1+v e\end{array}$ & 52.5 & 100 & Hossain et al., 2017 \\
\hline
\end{tabular}




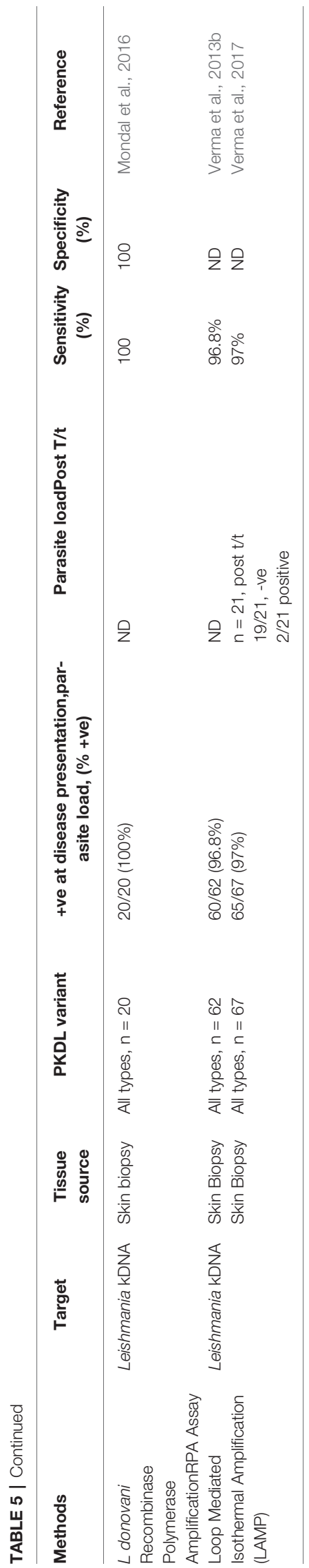

Verma et al., 2015). Several target sequences have been used for the PCR like ribosomal RNA genes, kinetoplast DNA (kDNA), mini-exonderived RNA (med RNA) genes and genomic repeats, the $\beta$-tubulin gene region, glycoprotein 63 (gp63) gene locus, internal transcribed spacer (ITS) regions and has been endorsed as a more sensitive method (Salotra et al., 2003 and references therein). Previously, a standardized real time PCR assay, based on Taqman chemistry that targeted the conserved REPL-repeat region of the Leishmania genome was used to assess the infection/disease dynamic in asymptomatic $L$. donovaniinfected individuals (Mondal et al., 2016, Table 5). Although kDNA based qPCR or real-time PCR has allowed for detection and quantification of the number of parasites at disease presentation (Table 5), it is equally important that longitudinal studies be performed to evaluate the qPCR as a tool to quantify the parasite clearance post-treatment (Ramesh et al., 2011; Verma et al., 2013a; Ramesh et al., 2015b; Bhargava et al., 2018; Moulik et al., 2018). Accordingly, we propose an algorithm for monitoring parasite kinetics (Figure 2).

Patients are enrolled if they present with clinical features suggestive of PKDL either by active case detection and reporting at a medical camp, or presenting in the Dermatology outpatient departments (OPD) of Govt. Medical Colleges. The suspected cases should be examined, and the rK39 strip test performed and if positive, a confirmation by ITS-1 PCR should be performed using a $4 \mathrm{~mm}$ skin biopsy (Das et al., 2011). In cases where rk39 is negative, but the patient provides a history of VL and/or clinical features are strongly suggestive of PKDL, ITS-1 PCR should be performed using DNA isolated from a skin biopsy. In cases that are ITS1-PCR positive, a kDNA based qPCR should be performed for determination of parasite load at disease presentation (Moulik et al., 2018). After completion of treatment as per guidelines of the National Vector Borne Disease Control Programme (NVBDCP), the parasite load should again be quantified by kDNA based qPCR (Moulik et al., 2018). Patients with a parasite load $<10$ parasites/ $\mu$ g genomic DNA may be considered as cured, and clinically followed up for possible reappearance of lesions. However, in patients with $>10$

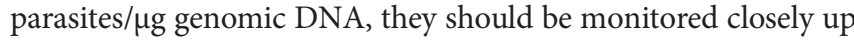
to 1 year and if the skin lesions increase, a repeat qPCR done. The parasite load that constitutes complete parasitological clearance remains a pertinent, yet unanswered question and it may be anticipated that implementation of such an algorithm may provide this information (Figure 2).

The nested PCR method targeted at minicircle kDNA of Leishmania proved to be highly effective and useful for detecting L. donovani genes in skin biopsy specimens from patients with PKDL (Nasreen et al., 2012). In PKDL, Mondal et al. (2016) demonstrated the diagnostic potential of the recombinase polymerase amplification (RPA) assay. This test was performed using a mobile suitcase laboratory approach, which endorsed its applicability in field settings (Table 5). However, its potential for monitoring the treatment outcome is warranted. Another fieldfriendly adaptation for DNA-based detection for PKDL cases is the (closed tube) loop-mediated isothermal application (LAMP, Verma et al., 2013b; Verma et al., 2017) but like the RPA assay, its efficacy for monitoring treatment remains to be assessed. 


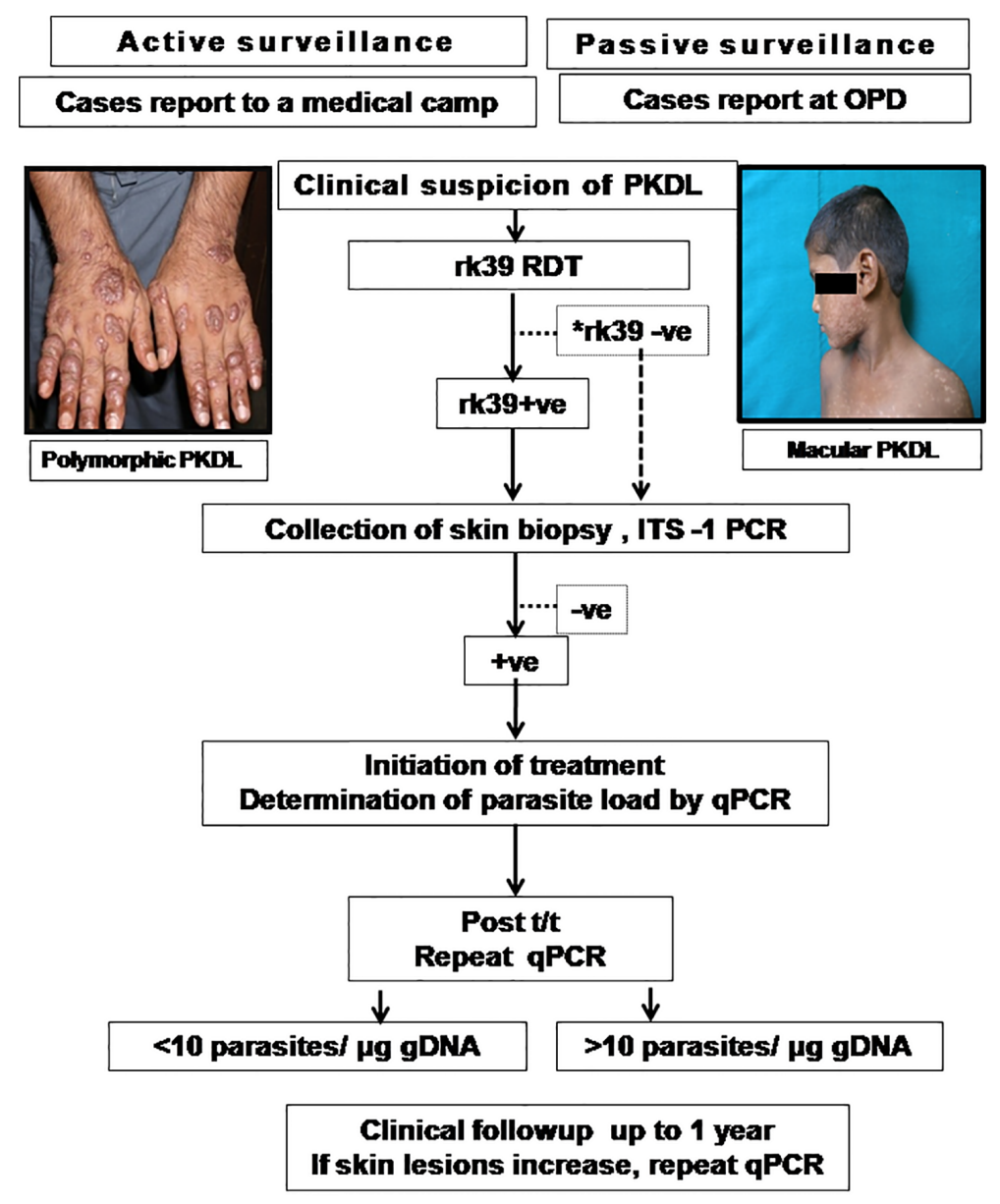

*If history of VL and/or clinical suspicion is strong

FIGURE 2 | Proposed algorithm for diagnosis of PKDL.

The availability of biomarkers and "test of cure" tools for Leishmaniasis will facilitate not only monitoring of active VL cases but also provide important epidemiological data. Therefore, it is necessary to establish an algorithm that can potentially differentiate between patients progressing to active VL vis-a-vis those that remain asymptomatic. Similarly, identification of biomarkers that can predict which cases of VL are likely to develop PKDL would be a value addition for the elimination program.

\section{Xenodiagnosis in VL and PKDL}

Xenodiagnosis is the classical approach for quantifying transmissibility from a host to an insect species with a view to distinguish infectious from non-infectious hosts (Singh et al., 2020). This method when applied can help define the characteristics of $L$. donovani transmission and provide key epidemiological evidence to guide the program. During xenodiagnosis of human VL caused by L. infantum, successful transmission to sand flies was confirmed in 6/6 (100\%) VL-HIV co-infected patients (Molina et al., 1999). Similarly, in a study in Brazil, 11/44 (25\%) VL patients were established as transmitters and importantly, no infections were detected in sand flies allowed to feed on 147 "asymptomatic" subjects (Costa et al., 2000). In an Indian study by Mukhopadhyay and Mishra (1991), one sandfly out of $183(0.5 \%)$ that fed on VL patients during the day time was infected, whereas in sandflies fed similarly at midnight, 4/75 (5\%) were infected, suggesting a periodicity for blood or tissue parasitemia.

In order to find a correlation, if any, between sandfly infection rates and parasite load, qPCR was performed using skin and blood sourced from patients with PKDL (Molina et al., 2017). It was further expanded to larger cohorts in Bangladesh wherein the infectiousness was higher in the polymorphic variant (17/21 81\%) vs. macular (9/35, 35\%) cases (Mondal et al., 2019); similarly, a substantial proportion of VL cases $(10 / 15,67 \%)$ were also able to transmit to sandflies (Mondal et al., 2019). Taken together, as tracking of the Leishmania parasite in sandflies provides key information regarding the infectiousness of VL and PKDL cases 
(Singh et al., 2020), application of molecular tools could provide information regarding the reservoir competence.

\section{DISCUSSION}

The availability and accessibility of new biomarkers and diagnostic tests for Leishmaniasis can facilitate not only the confirmation of active VL and PKDL cases, but also allow for epidemiological studies to be undertaken, identification of asymptomatic individuals, assess the degree of infectiousness to sand flies, and last but certainly not the least, monitor treatment efficacy in an objective and precise manner. It is necessary to establish an algorithm that differentiates between patients progressing to active VL vis-a-vis those that remain asymptomatic. Another aspect is the availability of markers for recognising patients with VL who develop PKDL, as this may have a considerable impact on disease control. The biggest hurdle so far is the monitoring of antileishmanial therapy and longitudinal studies are necessary to understand the parasite dynamics.

\section{CONCLUDING REMARKS}

In Leishmaniasis (particularly VL and PKDL), the biomarkers detailed in this review have been primarily used as diagnostic tools, and many of them have proven to be fairly robust and reproducible. In order to sustain the gains achieved via the kalaazar elimination program, the last mile strategies should include (1) validation of tools for monitoring anti-leishmanial therapy, (2) promoting epidemiological surveillance in the postelimination phase for detection of potential outbreaks, along with (3) supporting research focussed on identification of proxy

\section{REFERENCES}

Abass, E., Kang, C., Martinkovic, F., Semião-Santos, S. J., Sundar, S., Walden, P., et al. (2015). Heterogeneity of Leishmania donovani parasites complicates diagnosis of Visceral leishmaniasis: comparison of different serological tests in three endemic regions. PLoS One 10, e0116408. doi: 10.1371/journal.pone. 0116408

Abbasi, I., Aramin, S., Hailu, A., Shiferaw, W., Kassahun, A., Belay, S., et al. (2013). Evaluation of PCR procedures for detecting and quantifying Leishmania donovani DNA in large numbers of dried human blood samples from a Visceral Leishmaniasis focus in Northern Ethiopia. BMC Infect. Dis. 13:153. doi: 10.1186/1471-2334-13-153

Abbasi, I., Kirstein, O. D., Hailu, A., and Warburg, A. (2016). Optimization of loop-mediated isothermal amplification (LAMP) assays for the detection of Leishmania DNA in human blood samples. Acta Trop. 162, 20-26. doi: 10.1016/j.actatropica.2016.06.009

Abeijon, C., Alves, F., Monnerat, S., Wasunna, M., Mbui, J., Viana, A. G., et al. (2019). Development of a Multiplexed Assay for Detection of Leishmania donovani and Leishmania infantum Protein Biomarkers in Urine Samples of Patients with Visceral Leishmaniasis. J. Clin. Microbiol. 57, e02076-e02018. doi: 10.1128/JCM.02076-18

Abeijon, C., Alves, F., Monnerat, S., Mbui, J., Viana, A. G., Almeida, R. M., et al. (2020). Urine-based antigen detection assay for diagnosis of Visceral Leishmaniasis using monoclonal antibodies specific for six protein biomarkers of Leishmania infantum / Leishmania donovani. PLoS. Negl. Trop. Dis. 14, e0008246. doi: 10.1371/journal.pntd.0008246 markers for detecting sandfly infectivity (National Vector Borne Disease Control Programme, 2020).

\section{DATA AVAILABILITY STATEMENT}

The original contributions presented in the study are included in the article/supplementary material. Further inquiries can be directed to the corresponding author.

\section{AUTHOR CONTRIBUTIONS}

SM, SS, and MC performed the literature search, prepared, and edited the manuscript. All authors contributed to the article and approved the submitted version.

\section{FUNDING}

This study received financial support from Indian Council for Medical Research (Grant number: 6/9-7[151]2017-ECD II); Dept. of Health Research, Govt. of India (Grant number: DHR/HRD/Fellowship/SUG-05/2015-16); Fund for Improvement of S\&T infrastructure in Universities and Higher Educational Institutions (FIST) Program, Dept. of Science and Technology, Govt. of India (DST-FIST) (Grant number: SR/FST/ LS1-663/2016); Dept. of Science and Technology, Govt. of West Bengal (Grant number: 969 [Sanc.]/ST/P/S\&T/9G-22/2016). MC is a recipient of a JC Bose Fellowship (JCB/2019/000043), Science Engineering \& Research Board, DST, Govt. of India and SS a recipient of Junior Research Fellowship, CSIR, Govt. of India.

Adams, E. R., Jacquet, D., Schoone, G., Gidwani, K., Boelaert, M., and Cunningham, J. (2012). Leishmaniasis direct agglutination test: using pictorials as training materials to reduce inter-reader variability and improve accuracy. PLoS Negl. Trop. Dis. 6, e1946. doi: 10.1371/journal.pntd.0001946

Adams, E. R., Versteeg, I., and Leeflang, M. M. (2013). Systematic Review into Diagnostics for Post-Kala-Azar Dermal Leishmaniasis (PKDL). J. Trop. Med. 2013:150746. doi: 10.1155/2013/150746

Adams, E. R., Schoone, G., Versteeg, I., Gomez, M. A., Diro, E., Mori, Y., et al. (2018). Development and Evaluation of a Novel Loop-Mediated Isothermal Amplification Assay for Diagnosis of Cutaneous and Visceral Leishmaniasis. J. Clin. Microbiol. 56, e00386-e00318. doi: 10.1128/JCM. 00386-18

Akhoundi, B., Mohebali, M., Shojaee, S., Jalali, M., Kazemi, B., Bandehpour, M., et al. (2013). Rapid detection of human and canine Visceral Leishmaniasis: assessment of a latex agglutination test based on the A2 antigen from amastigote forms of Leishmania infantum. Exp. Parasitol 133, 307-313. doi: 10.1016/j.exppara

Alves, F., Bilbe, G., Blesson, S., Goyal, V., Monnerat, S., Mowbray, C., et al. (2018). Recent Development of Visceral Leishmaniasis Treatments: Successes, Pitfalls, and Perspectives. Clin. Microbiol. Rev. 31, e00048-e00018. doi: 10.1128/ CMR.00048-18

Anam, K., Afrin, F., Banerjee, D., Pramanik, N., Guha, S. K., Goswami, R. P., et al. (1999). Immunoglobulin subclass distribution and diagnostic value of Leishmania donovani antigen-specific immunoglobulin G3 in Indian kalaazar patients. Clin. Diagn. Lab. Immunol. 6, 231-235. doi: 10.1128/ CDLI.6.2.231-235.1999 
Ansari, N. A., Kumar, R., Raj, A., and Salotra, P. (2008). Elevated levels of IgG3 and IgG4 subclass in paediatric cases of kala azar. Parasite Immunol. 30, 403409. doi: $10.1111 / j .1365-3024.2008 .01036 . x$

Antinori, S., Calattini, S., Longhi, E., Bestetti, G., Piolini, R., Magni, C., et al. (2007). Clinical use of polymerase chain reaction performed on peripheral blood and bone marrow samples for the diagnosis and monitoring of Visceral Leishmaniasis in HIV-infected and HIV-uninfected patients: a single-center, 8year experience in Italy and review of the literature. Clin. Infect. Dis. 44, 16021610. doi: $10.1086 / 518167$

Bangert, M., Flores-Chávez, M. D., Llanes-Acevedo, I. P., Arcones, C., Chicharro, C., García, E., et al. (2018). Validation of rK39 immunochromatographic test and direct agglutination test for the diagnosis of Mediterranean Visceral Leishmaniasis in Spain. PLoS Negl. Trop. Dis. 12, e0006277. doi: 10.1371/ journal.pntd.0006277

Basiye, F. L., Mbuchi, M., Magiri, C., Kirigi, G., Deborggraeve, S., Schoone, G. J., et al. (2010). Sensitivity and specificity of the Leishmania OligoC-TesT and NASBA-oligochromatography for diagnosis of Visceral Leishmaniasis in Kenya. Trop. Med. Int. Health 15, 806-810. doi: 10.1111/j.1365-3156.2010.02548.x

Ben-Abid, M., Galaï, Y., Habboul, Z., Ben-Abdelaziz, R., Ben-Sghaier, I., Aoun, K., et al. (2017). Diagnosis of Mediterranean Visceral Leishmaniasis by detection of Leishmania-related antigen in urine and oral fluid samples. Acta Trop. 167, 71-72. doi: 10.1016/j.actatropica.2016.12.026

Bhargava, A., Ramesh, V., Verma, S., Salotra, P., and Bala, M. (2018). Revisiting the role of the slit-skin smear in the diagnosis of Indian post-kala-azar dermal Leishmaniasis. Indian J. Dermatol. Venereol. Leprol. 84, 690-695. doi: 10.4103/ ijdvl.IJDVL_970_16

Bhattacharyya, T., Ayandeh, A., Falconar, A. K., Sundar, S., El-Safi, S., Gripenberg, M. A., et al. (2014). IgG1 as a potential biomarker of post-chemotherapeutic relapse in Visceral Leishmaniasis, and adaptation to a rapid diagnostic test. PLoS Negl. Trop. Dis. 8, e3273. doi: 10.1371/journal.pntd.0003273

Boelaert, M., El-Safi, S., Hailu, A., Mukhtar, M., Rijal, S., Sundar, S., et al. (2008). Diagnostic tests for kala-azar: a multi-centre study of the freeze-dried DAT, rK39 strip test and KAtex in East Africa and the Indian subcontinent. Trans. $R$. Soc Trop. Med. Hyg. 102, 32-40. doi: 10.1016/j.trstmh.2007.09.003

Bourgeois, N., Lachaud, L., Reynes, J., Rouanet, I., Mahamat, A., and Bastien, P. (2008). Long-term monitoring of Visceral Leishmaniasis in patients with AIDS: relapse risk factors, value of polymerase chain reaction, and potential impact on secondary prophylaxis. J. Acquir. Immune. Defic. Syndr. 48, 13-19. doi: 10.1097/QAI.0b013e318166af5d

Burza, S., Croft, S. L., and Boelaert, M. (2018). Leishmaniasis. Lancet 392, 951-970. doi: $10.1016 /$ S0140-6736(18)31204-2

Cañavate, C., Herrero, M., Nieto, J., Cruz, I., Chicharro, C., Aparicio, P., et al. (2011). Evaluation of two rK39 dipstick tests, direct agglutination test, and indirect fluorescent antibody test for diagnosis of Visceral Leishmaniasis in a new epidemic site in highland Ethiopia. Am. J. Trop. Med. Hyg. 84, 102-106. doi: 10.4269/ajtmh.2011.10-0229

Chappuis, F., Rijal, S., Singh, R., Acharya, P., Karki, B. M., Das, M. L., et al. (2003). Prospective evaluation and comparison of the direct agglutination test and an rK39-antigen-based dipstick test for the diagnosis of suspected kala-azar in Nepal. Trop. Med. Int. Health 8, 277-285. doi: 10.1046/j.1365-3156.2003. 01026.x

Chatterjee, M., Basu, K., Basu, D., Bannerjee, D., Pramanik, N., Guha, S. K., et al. (1998). Distribution of IgG subclasses in antimonial unresponsive Indian kalaazar patients. Clin. Exp. Immunol. 114, 408-413. doi: 10.1046/j.13652249.1998.00752.x

Chava, A. K., Chatterjee, M., Sundar, S., and Mandal, C. (2002). Development of an assay for quantification of linkage-specific O-acetylated sialoglycans on erythrocytes; its application in Indian Visceral Leishmaniasis. J. Immunol. Methods 270, 1-10. doi: 10.1016/s0022-1759(02)00216-8

Costa, C. H., Gomes, R. B., Silva, M. R., Garcez, L. M., Ramos, P. K., Santos, R. S., et al. (2000). Competence of the human host as a reservoir for Leishmania chagasi. J. Infect. Dis. 182, 997-1000. doi: 10.1086/315795

Cota, G. F., de Sousa, M. R., Demarqui, F. N., and Rabello, A. (2012). The diagnostic accuracy of serologic and molecular methods for detecting Visceral Leishmaniasis in HIV infected patients: meta-analysis. PLoS Negl. Trop. Dis. 6, e1665. doi: 10.1371/journal.pntd.0001665

Cruz, I., Cañavate, C., Rubio, J. M., Morales, M. A., Chicharro, C., Laguna, F., et al. (2002). A nested polymerase chain reaction (Ln-PCR) for diagnosing and monitoring Leishmania infantum infection in patients co-infected with human immunodeficiency virus. Trans. R. Soc Trop. Med. Hyg. 96 Suppl 1, S185-S189. doi: 10.1016/s0035-9203(02)90074-x

da Silva, M., Brandão, N., Colovati, M., de Sousa, M., de Lima, L. C., Dorta, M. L., et al. (2018). Performance of two immunochromatographic tests for diagnosis of Visceral Leishmaniasis in patients coinfected with HIV. Parasitol. Res. 117, 419-427. doi: 10.1007/s00436-017-5716-3

Das, M. L., Deb, M., Karki, B. M., Sarif, M., Khanal, B., Bhattacharya, S. K., et al. (2007). Use of rK39 for diagnosis of post kala-azar dermal Leishmaniasis in Nepal. Southeast. Asian. J. Trop. Med. Public Health 38, 619-625.

Das, N. K., Singh, S. K., Ghosh, S., Sarkar, A., Mukhopadhyay, D., Roy, S., et al. (2011). Case series of misdiagnosis with rK39 strip test in Indian leishmaniasis. Am. J. Trop. Med. Hyg. 84, 688-691. doi: 10.4269/ajtmh.2011.10-0590

Das, V., Bimal, S., Siddiqui, N. A., Kumar, A., Pandey, K., Sinha, S. K., et al. (2020). Conversion of asymptomatic infection to symptomatic Visceral Leishmaniasis: A study of possible immunological markers. PLoS Negl. Trop. Dis. 14, e0008272. doi: 10.1371/journal.pntd.0008272

Datta, S., Modak, D., Sarkar, S., Saha, B., and Mukhopadhyay, S. (2015). Identification and glycobiological characterization of circulating immune complexes in patients with Visceral Leishmaniasis and post kala azar dermal Leishmaniasis. Indian J. Exp. Biol. 53, 321-328.

Datta, S., Ghosh, M., Sarkar, S., Saha, B., and Mukhopadhyay, S. (2019). Evaluation of anti-gal enzyme-linked immunosorbent assay for the diagnosis of Indian post-kala-azar dermal Leishmaniasis. Indian J. Dermatol. Venereol. Leprol. 85, 578-589. doi: 10.4103/ijdvl.IJDVL_485_18

Deborggraeve, S., Boelaert, M., Rijal, S., De Doncker, S., Dujardin, J. C., Herdewijn, P., et al. (2008). Diagnostic accuracy of a new Leishmania PCR for clinical Visceral Leishmaniasis in Nepal and its role in diagnosis of disease. Trop. Med. Int. Health 13, 1378-1383. doi: 10.1111/j.1365-3156.2008.02154.x

Deniau, M., Cañavate, C., Faraut-Gambarelli, F., and Marty, P. (2003). The biological diagnosis of Leishmaniasis in HIV-infected patients. Ann. Trop. Med. Parasitol. 97, 115-133. doi: 10.1179/000349803225002598

Dhom-Lemos, L., Viana, A. G., Cunha, J., Cardoso, M. S., Mendes, T., Pinheiro, G., et al. (2019). Leishmania infantum recombinant kinesin degenerated derived repeat (rKDDR): A novel potential antigen for serodiagnosis of Visceral Leishmaniasis. PLoS One 14, e0211719. doi: 10.1371/journal.pone.0211719

Dias, D. S., Ribeiro, P., Salles, B., Santos, T., Ramos, F. F., Lage, D. P., et al. (2018). Serological diagnosis and prognostic of tegumentary and Visceral Leishmaniasis using a conserved Leishmania hypothetical protein. Parasitol. Int. 67, 344-350. doi: 10.1016/j.parint.2018.02.001

Didwania, N., Ejazi, S. A., Chhajer, R., Sabur, A., Mazumder, S., Kamran, M., et al. (2020). Evaluation of Cysteine Protease C of Leishmania donovani in comparison with Glycoprotein 63 and Elongation Factor-1 $\alpha$ for diagnosis of human Visceral Leishmaniasis and for post-treatment follow-up response. J. Clin. Microbiol. 58, e00213. doi: 10.1128/JCM.00213-20

Diro, E., Techane, Y., Tefera, T., Assefa, Y., Kebede, T., Genetu, A., et al. (2007). Field evaluation of FD-DAT, rK39 dipstick and KATEX (urine latex agglutination) for diagnosis of Visceral Leishmaniasis in northwest Ethiopia. Trans. R. Soc Trop. Med. Hyg. 101, 908-914. doi: 10.1016/j.trstmh.2007.05.002

Dixit, K. K., Verma, S., Singh, O. P., Singh, D., Singh, A. P., Gupta, R., et al. (2018). Validation of SYBR green I based closed tube loop mediated isothermal amplification (LAMP) assay and simplified direct-blood-lysis (DBL)-LAMP assay for diagnosis of Visceral Leishmaniasis(VL). PLoS Negl. Trop. Dis. 12, e0006922. doi: 10.1371/journal.pntd.0006922

Duarte, M. C., Lage, D. P., Martins, V. T., Costa, L. E., Salles, B., Carvalho, A., et al. (2017). Performance of Leishmania braziliensis enolase protein for the serodiagnosis of canine and human Visceral leishmaniosis. Vet. Parasitol. 238, 77-81. doi: 10.1016/j.vetpar.2017.03.024

Duthie, M. S., Goto, Y., Ghosh, P., and Mondal, D. (2019). Impact of sequelae of Visceral Leishmaniasis and their contribution to ongoing transmission of Leishmania donovani. Pathog. Dis. 77, ftz057. doi: 10.1093/femspd/ftz057

Ejazi, S. A., Bhattacharya, P., Bakhteyar, M. A., Mumtaz, A. A., Pandey, K., Das, V. N., et al. (2016). Noninvasive Diagnosis of Visceral Leishmaniasis: Development and Evaluation of Two Urine-Based Immunoassays for Detection of Leishmania donovani Infection in India. PLoS Negl. Trop. Dis. 10, e0005035. doi: 10.1371/journal.pntd.0005035. eCollection 2016 Oct.

Ejazi, S. A., Ghosh, S., Saha, S., Choudhury, S. T., Bhattacharyya, A., Chatterjee, M., et al. (2019). A multicentric evaluation of dipstick test for serodiagnosis of 
Visceral Leishmaniasis in India, Nepal, Sri Lanka, Brazil, Ethiopia and Spain. Sci. Rep. 9, 9932. doi: 10.1038/s41598-019-46283-9

El Hassan, A. M., Khalil, E. A., Elamin, W. M., El Hassan, L. A., Ahmed, M. E., and Musa, A. M. (2013). Misdiagnosis and mistreatment of post-kala-azar dermal Leishmaniasis. Case Rep. Med. 2013:351579. doi: 10.1155/2013/351579

Fisa, R., Riera, C., Ribera, E., Gállego, M., and Portús, M. (2002). A nested polymerase chain reaction for diagnosis and follow-up of human Visceral Leishmaniasis patients using blood samples. Trans. R. Soc Trop. Med. Hyg. 96 Suppl 1, S191-S194. doi: 10.1016/s0035-9203(02)90075-1

Fonseca, A. M., Faria, A. R., Rodrigues, F. T., Nagem, R. A., Magalhães, R. D., Cunha, J. L., et al. (2014). Evaluation of three recombinant Leishmania infantum antigens in human and canine Visceral Leishmaniasis diagnosis. Acta Trop. 137, 25-30. doi: 10.1016/j.actatropica.2014.04.028

Freire, M. L., Assis, T., Avelar, D. M., Rabello, A., and Cota, G. (2018). Evaluation of a new brand of immunochromatographic test for Visceral Leishmaniasis in Brazil made available from 2018. Rev. Inst. Med. Trop. Sao Paulo. 60, e49. doi: $10.1590 / \mathrm{s} 1678-9946201860049$

Freire, M. L., Machado de Assis, T., Oliveira, E., Moreira de Avelar, D., Siqueira, I. C., Barral, A., et al. (2019). Performance of serological tests available in Brazil for the diagnosis of human Visceral Leishmaniasis. PLoS Negl. Trop. Dis. 13, e0007484. doi: 10.1371/journal.pntd.0007484

Ganguly, S., Das, N. K., Barbhuiya, J. N., and Chatterjee, M. (2010). Post-kala-azar dermal leishmaniasis-an overview. Int. J. Dermatol. 49, 921-931. doi: 10.1111/ j.1365-4632.2010.04558.x

Gao, C. H., Yang, Y. T., Shi, F., Wang, J. Y., Steverding, D., and Wang, X. (2015). Development of an Immunochromatographic Test for Diagnosis of Visceral Leishmaniasis Based on Detection of a Circulating Antigen. PLoS Negl. Trop. Dis. 9, e0003902. doi: 10.1371/journal.pntd.0003902

Gedda, M. R., Singh, B., Kumar, D., Singh, A. K., Madhukar, P., Upadhyay, S., et al. (2020). Post kala-azar dermal Leishmaniasis: A threat to elimination program. PLoS Negl. Trop. Dis. 14, e0008221. doi: 10.1371/journal.pntd.0008221

Ghasemian, M., Gharavi, M. J., Akhlaghi, L., Mohebali, M., Meamar, A. R., Aryan, E., et al. (2014). Development and Assessment of Loop-Mediated Isothermal Amplification (LAMP) Assay for the Diagnosis of Human Visceral Leishmaniasis in Iran. Iran J. Parasitol. 9, 50-59.

Ghosh, P., Bhaskar, K. R., Hossain, F., Khan, M. A., Vallur, A. C., Duthie, M. S., et al. (2016). Evaluation of diagnostic performance of rK28 ELISA using urine for diagnosis of Visceral Leishmaniasis. Parasitol. Vectors. 9, 383. doi: 10.1186/ s13071-016-1667-2

Ghosh, P., Hasnain, M. G., Hossain, F., Khan, M., Chowdhury, R., Faisal, K., et al. (2018). Evaluation of Real-time PCR for Diagnosis of Post-Kala-azar Dermal Leishmaniasis in Endemic Foci of Bangladesh. Open Forum Infect. Dis. 5, 234. doi: $10.1093 /$ ofid/ofy234

Hailu, A., and Berhe, N. (2002). The performance of direct agglutination tests (DAT) in the diagnosis of Visceral Leishmaniasis among Ethiopian patients with HIV co-infection. Ann. Trop. Med. Parasitol. 96, 25-30. doi: 10.1179/ 000349802125000475

Hirve, S., Kroeger, A., Matlashewski, G., Mondal, D., Banjara, M. R., Das, P., et al. (2017). Towards elimination of Visceral Leishmaniasis in the Indian subcontinent-Translating research to practice to public health. PLoS Negl. Trop. Dis. 11, e0005889. doi: 10.1371/journal.pntd.0005889

Hossain, F., Ghosh, P., Khan, M., Duthie, M. S., Vallur, A. C., Picone, A., et al. (2017). Real-time PCR in detection and quantitation of Leishmania donovani for the diagnosis of Visceral Leishmaniasis patients and the monitoring of their response to treatment. PLoS One 12, e0185606. doi: 10.1371/journal.pone. 0185606

Hosseini Farash, B. R., Mohebali, M., Kazemi, B., Hajjaran, H., Fata, A., Raoofian, R., et al. (2020). The rK39 Antigen from an Iranian Strain of Leishmania infantum: Detection of Anti-Leishmania Antibodies in Humans and Dogs. Iran J. Parasitol. 15, 48-56.

Hu, X. S., Yang, W. T., Lu, H. G., Yan, H. P., Cheng, J. P., Ma, Y., et al. (2000). Sequencing a specific kinetoplast DNA fragment of Leishmania donovani for polymerase chain reaction amplification in diagnosis of Leishmaniasis in bone marrow and blood samples. J. Parasitol. 86, 822-826. doi: 10.1645/0022-3395 (2000)086[0822:SASKDF]2.0.CO;2

Humbert, M. V., Costa, L. E., Katis, I., Fonseca Ramos, F., Sanchéz Machado, A., Sones, C., et al. (2019). A rapid diagnostic test for human Visceral Leishmaniasis using novel Leishmania antigens in a Laser Direct-Write
Lateral Flow Device. Emerg. Microbes Infect. 8, 1178-1185. doi: 10.1080/ 22221751.2019.1635430

Jaiswal, P., Datta, S., Sardar, B., Chaudhuri, S. J., Maji, D., Ghosh, M., et al. (2018). Glycoproteins in circulating immune complexes are biomarkers of patients with Indian PKDL: A study from endemic districts of West Bengal, India. PloS One 13, e0192302. doi: 10.1371/journal.pone.0192302

Khan, M. G., Bhaskar, K. R., Salam, M. A., Akther, T., Pluschke, G., and Mondal, D. (2012). Diagnostic accuracy of loop-mediated isothermal amplification (LAMP) for detection of Leishmania DNA in buffy coat from Visceral Leishmaniasis patients. Parasitol. Vectors. 5:280. doi: 10.1186/1756-3305-5-280

Kip, A. E., Balasegaram, M., Beijnen, J. H., Schellens, J. H., de Vries, P. J., and Dorlo, T. P. (2015). Systematic review of biomarkers to monitor therapeutic response in Leishmaniasis. Antimicrob. Agents. Chemother. 59, 1-14. doi: 10.1128/AAC.04298-14

Kip, A. E., Wasunna, M., Alves, F., Schellens, J., Beijnen, J. H., Musa, A. M., et al. (2018). Macrophage Activation Marker Neopterin: A Candidate Biomarker for Treatment Response and Relapse in Visceral Leishmaniasis. Front. Cell. Infect. Microbiol. 8, 181. doi: 10.3389/fcimb.2018.00181

Kumar, S., Kumar, D., Chakravarty, J., Rai, M., and Sundar, S. (2012). Identification and characterization of a novel Leishmania donovani antigen for serodiagnosis of Visceral Leishmaniasis. Am. J. Trop. Med. Hyg. 86, 601605. doi: 10.4269/ajtmh.2012.11-0261

Lachaud, L., Dereure, J., Chabbert, E., Reynes, J., Mauboussin, J. M., Oziol, E., et al. (2000). Optimized PCR using patient blood samples for diagnosis and followup of Visceral Leishmaniasis, with special reference to AIDS patients. J. Clin. Microbiol. 38, 236-240. doi: 10.1128/JCM.38.1.236-240.2000

Lindoso, J., Moreira, C., Cunha, M. A., and Queiroz, I. T. (2018). Visceral Leishmaniasis and HIV coinfection: current perspectives. HIV/AIDS 10, 193-201. doi: 10.2147/HIV.S143929

Machado de Assis, T. S., Rabello, A., and Werneck, G. L. (2012). Latent class analysis of diagnostic tests for Visceral Leishmaniasis in Brazil. Trop. Med. Int. Health 17, 1202-1207. doi: 10.1111/j.1365-3156.2012.03064.x

Machado, A. S., Ramos, F. F., Oliveira-da-Silva, J. A., Santos, T., Ludolf, F., Tavares, G., et al. (2020). A Leishmania infantum hypothetical protein evaluated as a recombinant protein and specific B-cell epitope for the serodiagnosis and prognosis of Visceral Leishmaniasis. Acta Trop. 203:105318. doi: 10.1016/j.actatropica.2019.105318

Mandal, J., Khurana, S., Dubey, M. L., Bhatia, P., Varma, N., and Malla, N. (2008). Evaluation of direct agglutination test, rk39 Test, and ELISA for the diagnosis of Visceral Leishmaniasis. Am. J. Trop. Med. Hyg. 79, 76-78. doi: 10.4269/ ajtmh.2008.79.76

Mary, C., Faraut, F., Lascombe, L., and Dumon, H. (2004). Quantification of Leishmania infantum DNA by a real-time PCR assay with high sensitivity. J. Clin. Microbiol. 42, 5249-5255. doi: 10.1128/JCM.42.11.5249-5255.2004

Mary, C., Faraut, F., Drogoul, M. P., Xeridat, B., Schleinitz, N., Cuisenier, B., et al. (2006). Reference values for Leishmania infantum parasitemia in different clinical presentations: quantitative polymerase chain reaction for therapeutic monitoring and patient follow-up. Am. J. Trop. Med. Hyg. 75, 858-863. doi: 10.4269/ajtmh.2006.75.858

Mathur, P., Samantaray, J. C., Vajpayee, M., and Samanta, P. (2006). Visceral Leishmaniasis/human immunodeficiency virus co-infection in India: the focus of two epidemics. J. Med. Microbiol. 55, 919-922. doi: 10.1099/jmm. 0.46574-0

Matlashewski, G., Das, V. N., Pandey, K., Singh, D., Das, S., Ghosh, A. K., et al. (2013). Diagnosis of Visceral Leishmaniasis in Bihar India: comparison of the rK39 rapid diagnostic test on whole blood versus serum. PLoS Negl. Trop. Dis. 7, e2233. doi: 10.1371/journal.pntd.0002233

Mbui, J., Wasunna, M., Balasegaram, M., Laussermayer, A., Juma, R., Njenga, S. N., et al. (2013). Validation of two rapid diagnostic tests for Visceral Leishmaniasis in Kenya. PLoS Negl. Trop. Dis. 7, e2441. doi: 10.1371/journal.pntd.0002441

Medeiros, F. A., Gomes, L. I., Oliveira, E., de Souza, C. S., Mourão, M. V., Cota, G. F., et al. (2017). Development and Validation of a PCR-ELISA for the Diagnosis of Symptomatic and Asymptomatic Infection by Leishmania (Leishmania) infantum. J. Trop. Med. 2017:7364854. doi: 10.1155/2017/7364854

Mehrotra, S., Fakiola, M., Oommen, J., Jamieson, S. E., Mishra, A., Sudarshan, M., et al. (2011). Genetic and functional evaluation of the role of CXCR1 and CXCR2 in susceptibility to Visceral Leishmaniasis in north-east India. BMC Med. Genet. 12:162. doi: 10.1186/1471-2350-12-162 
Molina, R., Lohse, J. M., Pulido, F., Laguna, F., López-Vélez, R., and Alvar, J. (1999). Infection of sand flies by humans coinfected with Leishmania infantum and human immunodeficiency virus. Am. J. Trop. Med. Hyg. 60, 51-53. doi: 10.4269/ajtmh.1999.60.51

Molina, I., Fisa, R., Riera, C., Falcó, V., Elizalde, A., Salvador, F., et al. (2013). Ultrasensitive real-time PCR for the clinical management of Visceral Leishmaniasis in HIV-Infected patients. Am. J. Trop. Med. Hyg. 89, 105-110. doi: 10.4269/ajtmh.12-0527

Molina, R., Ghosh, D., Carrillo, E., Monnerat, S., Bern, C., Mondal, D., et al. (2017). Infectivity of Post-Kala-azar Dermal Leishmaniasis Patients to Sand Flies: Revisiting a Proof of Concept in the Context of the Kala-azar Elimination Program in the Indian Subcontinent. Clin. Infect. Dis. 65, 150-153. doi: $10.1093 / \mathrm{cid} / \mathrm{cix} 245$

Mondal, D., Nasrin, K. N., Huda, M. M., Kabir, M., Hossain, M. S., Kroeger, A., et al. (2010). Enhanced case detection and improved diagnosis of PKDL in a Kala-azar-endemic area of Bangladesh. PLoS Negl. Trop. Dis. 4, e832. doi: 10.1371/journal.pntd.0000832

Mondal, D., Ghosh, P., Khan, M. A., Hossain, F., Böhlken-Fascher, S., Matlashewski, G., et al. (2016). Mobile suitcase laboratory for rapid detection of Leishmania donovani using recombinase polymerase amplification assay. Parasitol. Vectors. 9, 281. doi: 10.1186/s13071-016-1572-8

Mondal, D., Bern, C., Ghosh, D., Rashid, M., Molina, R., Chowdhury, R., et al. (2019). Quantifying the Infectiousness of Post-Kala-Azar Dermal Leishmaniasis Toward Sand Flies. Clin. Infect. Dis. 69, 251-258. doi: 10.1093/cid/ciy891

Moulik, S., Chaudhuri, S. J., Sardar, B., Ghosh, M., Saha, B., Das, N. K., et al. (2018). Monitoring of Parasite Kinetics in Indian Post-Kala-azar Dermal Leishmaniasis. Clin. Infect. Dis. 66, 404-410. doi: 10.1093/cid/cix808

Moura, A. S., Lopes, H. M., Mourão, M. V., and Morais, M. H. (2013). Performance of a rapid diagnostic test for the detection of Visceral Leishmaniasis in a large urban setting. Rev. Soc Bras. Med. Trop. 46, 589593. doi: 10.1590/0037-8682-0145-2013

Mukhopadhyay, A. K., and Mishra, R. N. (1991). Development of Leishmania donovani in Phlebotomus argentipes \& Ph. Papatasi Fed. Kala-azar Patients Bihar. Indian J. Med. Res. 93, 152-154.

Mukhopadhyay, D., Das, N. K., De Sarkar, S., Manna, A., Ganguly, D. N., Barbhuiya, J. N., et al. (2012). Evaluation of serological markers to monitor the disease status of Indian post kala-azar dermal Leishmaniasis. Trans. R. Soc Trop. Med. Hyg. 106, 668-676. doi: 10.1016/j.trstmh.2012. 07.005.

Mukhtar, M., Abdoun, A., Ahmed, A. E., Ghalib, H., Reed, S. G., Boelaert, M., et al. (2015). Diagnostic accuracy of rK28-based immunochromatographic rapid diagnostic tests for Visceral Leishmaniasis: a prospective clinical cohort study in Sudan. Trans. R. Soc Trop. Med. Hyg. 109, 594-600. doi: 10.1093/trstmh/trv060

Mukhtar, M., Ali, S. S., Boshara, S. A., Albertini, A., Monnerat, S., Bessell, P., et al. (2018). Sensitive and less invasive confirmatory diagnosis of Visceral Leishmaniasis in Sudan using loop-mediated isothermal amplification (LAMP). PLoS Negl. Trop. Dis. 12, e0006264. doi: 10.1371/journal.pntd. 0006264

Nasreen, S. A., Hossain, M. A., Paul, S. K., Mahmud, M. C., Ahmed, S., Ghosh, S., et al. (2012). PCR-based detection of Leishmania DNA in skin samples of post kala-azar dermal Leishmaniasis patients from an endemic area of Bangladesh. Jpn. J. Infect. Dis. 65, 315-317. doi: 10.7883/yoken.65.315

National Vector Borne Disease Control Programme (2020). Independent Assessment of Kala-azar Elimination Programme India. India, 9-20 ${ }^{\text {th }}$ Dec 2019. Available at: https://nvbdcp.gov.in/index4.php?lang=1\&level=0\&linkid= 469\&lid=3752 (Accessed August 25, 2020).

Notomi, T., Okayama, H., Masubuchi, H., Yonekawa, T., Watanabe, K., Amino, N., et al. (2000). Loop-mediated isothermal amplification of DNA. Nucleic Acids Res. 28, E63. doi: 10.1093/nar/28.12.e63

Nzelu, C. O., Kato, H., and Peters, N. C. (2019). Loop-mediated isothermal amplification (LAMP): An advanced molecular point-of-care technique for the detection of Leishmania infection. PLoS Negl. Trop. Dis. 13, e0007698. doi: 10.1371/journal.pntd.0007698

Oliveira, E., Saliba, S. W., Saliba, J. W., and Rabello, A. (2013). Validation of a direct agglutination test prototype kit for the diagnosis of Visceral Leishmaniasis. Trans. R. Soc Trop. Med. Hyg. 107, 243-247. doi: 10.1093/trstmh/trt004

Pita-Pereira, D., Lins, R., Oliveira, M. P., Lima, R. B., Pereira, B. A., Moreira, O. C., et al. (2012). SYBR Green-based real-time PCR targeting kinetoplast DNA can be used to discriminate between the main etiologic agents of Brazilian cutaneous and Visceral leishmaniases. Parasitol. Vectors. 5:15. doi: 10.1186/ 1756-3305-5-15

Portela, Á, Costa, L. E., Salles, B., Lima, M. P., Santos, T., Ramos, F. F., et al. (2018). Identification of immune biomarkers related to disease progression and treatment efficacy in human Visceral Leishmaniasis. Immunobiology 223, 303-309. doi: 10.1016/j.imbio.2017.10.043

Ramesh, V., Katara, G. K., Verma, S., and Salotra, P. (2011). Miltefosine as an effective choice in the treatment of post-kala-azar dermal Leishmaniasis. Br. J. Dermatol. 165, 411-414. doi: 10.1111/j.1365-2133.2011.10402.x

Ramesh, V., Kaushal, H., Mishra, A. K., Singh, R., and Salotra, P. (2015a). Clinicoepidemiological analysis of Post kala-azar dermal Leishmaniasis (PKDL) cases in India over last two decades: a hospital based retrospective study. BMC Public Health 15, 1092. doi: 10.1186/s12889-015-2424-8

Ramesh, V., Singh, R., Avishek, K., Verma, A., Deep, D. K., Verma, S., et al. (2015b). Decline in Clinical Efficacy of Oral Miltefosine in Treatment of Post Kala-azar Dermal Leishmaniasis (PKDL) in India. PLoS Negl. Trop. Dis. 9, e0004093. doi: 10.1371/journal.pntd.0004093

Redhu, N. S., Dey, A., Balooni, V., and Singh, S. (2006). Leishmania-HIV coinfection: an emerging problem in India. AIDS 20, 1213-1215. doi: 10.1097/ 01.aids.0000226971.42622.4e

Rezaei, Z., Van Reet, N., Pouladfar, G., Kühne, V., Ramezani, A., Sarkari, B., et al. (2019). Expression of a rK39 homologue from an Iranian Leishmania infantum isolate in Leishmania tarentolae for serodiagnosis of Visceral Leishmaniasis. Parasitol. Vectors. 12, 593. doi: 10.1186/s13071-019-3839-3

Riera, C., Fisa, R., Udina, M., Gállego, M., and Portus, M. (2004). Detection of Leishmania infantum cryptic infection in asymptomatic blood donors living in an endemic area (Eivissa, Balearic Islands, Spain) by different diagnostic methods. Trans. R. Soc Trop. Med. Hyg. 98, 102-110. doi: 10.1016/s0035-9203(03)00015-4

Rijal, S., Sundar, S., Mondal, D., Das, P., Alvar, J., and Boelaert, M. (2019). Eliminating Visceral Leishmaniasis in South Asia: the road ahead. BMJ 364: k5224. doi: 10.1136/bmj.k5224

Saad, A. A., Ahmed, N. G., Osman, O. S., Al-Basheer, A. A., Hamad, A., Deborggraeve, S., et al. (2010). Diagnostic accuracy of the Leishmania OligoC-TesT and NASBAOligochromatography for diagnosis of Leishmaniasis in Sudan. PLoS Negl. Trop. Dis. 4, e776. doi: 10.1371/journal.pntd. 0000776.

Saha, S., Mazumdar, T., Anam, K., Ravindran, R., Bairagi, B., Saha, B., et al. (2005). Leishmania promastigote membrane antigen-based enzyme-linked immunosorbent assay and immunoblotting for differential diagnosis of Indian post-kala-azar dermal Leishmaniasis. J. Clin. Microbiol. 43, 12691277. doi: 10.1128/JCM.43.3.1269-1277.2005

Salam, M. A., Khan, M. G., and Mondal, D. (2011). Urine antigen detection by latex agglutination test for diagnosis and assessment of initial cure of Visceral Leishmaniasis. Trans. R. Soc Trop. Med. Hyg. 105, 269-272. doi: 10.1016/ j.trstmh.2010.12.007

Salotra, P., Raina, A., and Negi, N. S. (1999). Immunoblot analysis of the antibody response to antigens of Leishmania donovani in Indian kala-azar. Br. J. Biomed. Sci. 56, 263-267.

Salotra, P., Sreenivas, G., Pogue, G. P., Lee, N., Nakhasi, H. L., Ramesh, V., et al. (2001). Development of a species-specific PCR assay for detection of Leishmania donovani in clinical samples from patients with kala-azar and post-kala-azar dermal Leishmaniasis. J. Clin. Microbiol. 39, 849-854. doi: 10.1128/JCM.39.3.849-854.2001

Salotra, P., Sreenivas, G., Beena, K. R., Mukherjee, A., and Ramesh, V. (2003). Parasite detection in patients with post kala-azar dermal Leishmaniasis in India: a comparison between molecular and immunological methods. J. Clin. Pathol. 56, 840-843. doi: 10.1136/jcp.56.11.840

Sanchez, M., Celeste, B. J., Lindoso, J., Fujimori, M., de Almeida, R. P., Fortaleza, C., et al. (2020). Performance of rK39-based immunochromatographic rapid diagnostic test for serodiagnosis of Visceral Leishmaniasis using whole blood, serum and oral fluid. PLoS One 15, e0230610. doi: 10.1371/ journal.pone.0230610

Santos, T., Cardoso, M. S., Machado, A. S., Siqueira, W. F., Ramos, F. F., Oliveirada-Silva, J. A., et al. (2019). Recombinant Leishmania eukaryotic elongation factor-1 beta protein: A potential diagnostic antigen to detect tegumentary and Visceral Leishmaniasis in dogs and humans. Microb. Pathog. 137:103783. doi: 10.1016/j.micpath.2019.103783

Santos-Gomes, G., Gomes-Pereira, S., Campino, L., Araújo, M. D., and Abranches, P. (2000). Performance of immunoblotting in diagnosis of Visceral Leishmaniasis in 
human immunodeficiency virus-Leishmania sp--coinfected patients. J. Clin. Microbiol. 38, 175-178. doi: 10.1128/JCM.38.1.175-178.2000

Sengupta, R., Chaudhuri, S. J., Moulik, S., Ghosh, M. K., Saha, B., Das, N. K., et al. (2019). Active surveillance identified a neglected burden of macular cases of Post Kala-azar Dermal Leishmaniasis in West Bengal. PLoS Negl. Trop. Dis. 13, e0007249. doi: 10.1371/journal.pntd.0007249

Singh, R., Subba Raju, B. V., Jain, R. K., and Salotra, P. (2005). Potential of direct agglutination test based on promastigote and amastigote antigens for serodiagnosis of post-kala-azar dermal Leishmaniasis. Clin. Diagn. Lab. Immunol. 12, 1191-1194. doi: 10.1128/CDLI.12.10.1191-1194.2005

Singh, D. P., Sundar, S., and Mohapatra, T. M. (2009). The rK39 strip test is nonpredictor of clinical status for kala-azar. BMC Res. Notes 2:187. doi: 10.1186/ 1756-0500-2-187

Singh, A., Ramesh, V., and Ramam, M. (2015). Histopathological characteristics of post kala-azar dermal Leishmaniasis: a series of 88 patients. Indian J. Dermatol. Venereol. Leprol. 81, 29-34. doi: 10.4103/0378-6323.148562

Singh, O. P., Hasker, E., Boelaert, M., Sacks, D., and Sundar, S. (2020). Xenodiagnosis to address key questions in Visceral Leishmaniasis control and elimination. PLoS Negl. Trop. Dis. 14, e0008363. doi: 10.1371/journal.pntd.0008363

Sinha, P. K., Bimal, S., Singh, S. K., Pandey, K., Gangopadhyay, D. N., and Bhattacharya, S. K. (2006). Pre- \& post-treatment evaluation of immunological features in Indian Visceral Leishmaniasis (VL) patients with HIV co-infection. Indian J. Med. Res. 123, 197-202.

Sreenivas, G., Ansari, N. A., Kataria, J., and Salotra, P. (2004). Nested PCR assay for detection of Leishmania donovani in slit aspirates from post-kala-azar dermal Leishmaniasis Lesions. J. Clin. Microbiol. 42, 1777-1778. doi: 10.1128/ jcm.42.4.1777-1778.2004

Srivastava, P., Mehrotra, S., Tiwary, P., Chakravarty, J., and Sundar, S. (2011). Diagnosis of Indian Visceral Leishmaniasis by nucleic acid detection using PCR. PLoS One 6, e19304. doi: 10.1371/journal.pone.0019304

Sudarshan, M., Weirather, J. L., Wilson, M. E., and Sundar, S. (2011). Study of parasite kinetics with antileishmanial drugs using real-time quantitative PCR in Indian Visceral Leishmaniasis. J. Antimicrob. Chemother. 66, 1751-1755. doi: $10.1093 / \mathrm{jac} / \mathrm{dkr} 185$

Sudarshan, M., Singh, T., Chakravarty, J., and Sundar, S. (2015). A correlative study of splenic parasite score and peripheral blood parasite load estimation by quantitative PCR in Visceral Leishmaniasis. J. Clin. Microbiol. 3, 3905-3907. doi: 10.1128/JCM.01465-15

Sundar, S., and Rai, M. (2002). Laboratory diagnosis of Visceral Leishmaniasis. Clin. Diagn. Lab. Immunol. 9, 951-958. doi: 10.1128/cdli.9.5.951-958.2002

Sundar, S., Singh, R. K., Maurya, R., Kumar, B., Chhabra, A., Singh, V., et al. (2006). Serological diagnosis of Indian Visceral Leishmaniasis: direct agglutination test versus rK39 strip test. Trans. R. Soc Trop. Med. Hyg. 100, 533-537. doi: 10.1016/j.trstmh.2005.08.018

Sundar, S., Singh, R. K., Bimal, S. K., Gidwani, K., Mishra, A., Maurya, R., et al. (2007). Comparative evaluation of parasitology and serological tests in the diagnosis of Visceral Leishmaniasis in India: a phase III diagnostic accuracy study. Trop. Med. Int. Health 12, 284-289. doi: 10.1111/j.1365-3156.2006.01775.x

Sundar, S., Singh, O. P., and Chakravarty, J. (2018). Visceral Leishmaniasis elimination targets in India, strategies for preventing resurgence. Expert Rev. Anti Infect. Ther. 16, 805-812. doi: 10.1080/14787210.2018.1532790

ter Horst, R., Tefera, T., Assefa, G., Ebrahim, A. Z., Davidson, R. N., and Ritmeijer, K. (2009). Field evaluation of rK39 test and direct agglutination test for diagnosis of Visceral Leishmaniasis in a population with high prevalence of human immunodeficiency virus in Ethiopia. Am. J. Trop. Med. Hyg. 80, 929-934. doi: 10.4269/aitmh.2009.80.929

Topno, R. K., Shankar, V., Dikhit, M. R., Madhukar, M., Pandey, K., Das, V., et al. (2018). Noninvasive Sweat-Based Diagnosis of Visceral Leishmaniasis and Post Kala-Azar Dermal Leishmaniasis. Am. J. Trop. Med. Hyg. 99, 1162-1164. doi: 10.4269/ajtmh.17-0749

Vaish, M., Bhatia, A., Reed, S. G., Chakravarty, J., and Sundar, S. (2012). Evaluation of rK28 antigen for serodiagnosis of Visceral Leishmaniasis in India. Clin. Microbiol. Infect. 18, 81-85. doi: 10.1111/j.1469-0691.2011.03540.x
Verma, S., Kumar, R., Katara, G. K., Singh, L. C., Negi, N. S., Ramesh, V., et al. (2010). Quantification of parasite load in clinical samples of Leishmaniasis patients: IL-10 level correlates with parasite load in Visceral Leishmaniasis. PLoS One 5, e10107. doi: 10.1371/journal.pone.0010107

Verma, S., Bhandari, V., Avishek, K., Ramesh, V., and Salotra, P. (2013a). Reliable diagnosis of post-kala-azar dermal Leishmaniasis (PKDL) using slit aspirate specimen to avoid invasive sampling procedures. Trop. Med. Int. Health 18, 268-275. doi: 10.1111/tmi.12047

Verma, S., Avishek, K., Sharma, V., Negi, N. S., Ramesh, V., and Salotra, P. (2013b). Application of loop-mediated isothermal amplification assay for the sensitive and rapid diagnosis of Visceral Leishmaniasis and post-kala-azar dermal Leishmaniasis. Diagn. Microbiol. Infect. $m$ Dis. 75, 390-395. doi: 10.1016/j. diagmicrobio.2013.01.011.

Verma, N., Bimal, S., Das, V. N., Pandey, K., Singh, D., Lal, C. S., et al. (2015). Clinicopathological and Immunological Changes in Indian Post Kala-Azar Dermal Leishmaniasis (PKDL) Cases in relation to Treatment: A Retrospective Study. Biomed. Res. Int. 2015:745062. doi: 10.1155/2015/745062

Verma, S., Singh, R., Sharma, V., Bumb, R. A., Negi, N. S., Ramesh, V., et al. (2017). Development of a rapid loop-mediated isothermal amplification assay for diagnosis and assessment of cure of Leishmania infection. BMC Infect. Dis. 17, 223. doi: 10.1186/s12879-017-2318-8

Vijayamahantesh, A. A., Dikhit, M. R., Pandey, R. K., Singh, K., Mishra, R., Das, V. N., et al. (2016). Elevated Serum ADA Activity as a Marker for Diagnosis and Prognosis of Visceral Leishmaniasis and Post Kala-Azar Dermal Leishmaniasis in Indian Patients. PLoS One 11, e0154117. doi: 10.1371/journal. pone. 0154117

Vink, M., Nahzat, S. M., Rahimi, H., Buhler, C., Ahmadi, B. A., Nader, M., et al. (2018). Evaluation of point-of-care tests for cutaneous Leishmaniasis diagnosis in Kabul, Afghanistan. EBioMedicine 37, 453-460. doi: 10.1016/j.ebiom.2018. 10.063

World Health Organization (2016). Process of validation of elimination of kalaazar as a public health problem (Accessed June 10, 2020). Available at: https:// www.who.int/Leishmaniasis/resources/Process_of_validation_of_VL_ elimination_SEA_CD_321.pdf.

World Health Organization (2020). Eliminating Visceral leishmaniasis: India takes decisive steps to overcome last-mile challenges. Available at: https:// www.who.int/news/item/05-03-2020-VL-India-takes-decisive-stepsovercome-last-mile-challenges (Accessed June 10, 2020).

Zhang, W. W., Ghosh, A. K., Mohamath, R., Whittle, J., Picone, A., Lypaczewski, P., et al. (2018). Development of a sandwich ELISA to detect Leishmania 40 S ribosomal protein S12 antigen from blood samples of Visceral Leishmaniasis patients. BMC Infect. Dis. 18, 500. doi: 10.1186/s12879-0183420-2

Zijlstra, E. E., Musa, A. M., Khalil, E. A., el-Hassan, I. M., and el-Hassan, A. M. (2003). Post-kala-azar dermal Leishmaniasis. Lancet Infect. Dis. 3, 87-98. doi: 10.1016/s1473-3099(03)00517-6

Zijlstra, E. E., Alves, F., Rijal, S., Arana, B., and Alvar, J. (2017). Post-kala-azar dermal Leishmaniasis in the Indian subcontinent: A threat to the South-East Asia Region Kala-azar Elimination Programme. PLoS Negl. Trop. Dis. 11, e0005877. doi: 10.1371/journal.pntd.0005877

Zijlstra, E. E. (2019). Biomarkers in Post-kala-azar Dermal Leishmaniasis. Front. Cell. Infect. Microbiol. 9:228. doi: 10.3389/fcimb.2019.00228

Conflict of Interest: The authors declare that the research was conducted in the absence of any commercial or financial relationships that could be construed as a potential conflict of interest.

Copyright (๑) 2021 Moulik, Sengupta and Chatterjee. This is an open-access article distributed under the terms of the Creative Commons Attribution License (CC BY). The use, distribution or reproduction in other forums is permitted, provided the original author(s) and the copyright owner(s) are credited and that the original publication in this journal is cited, in accordance with accepted academic practice. No use, distribution or reproduction is permitted which does not comply with these terms. 